\title{
NONLINEAR ANALYSIS OF BONDED COMPOSITE SINGLE-LAP JOINTS
}

\author{
E. Oterkus, ${ }^{*}$ A. Barut, ${ }^{\dagger}$ E. Madenci ${ }^{\dagger}$ \\ The University of Arizona, Tucson, AZ 85721 \\ S.S. Smeltzer III, ${ }^{\S}$ D.R. Ambur $₫$ \\ NASA Langley Research Center, Hampton, VA 23681
}

\begin{abstract}
$\underline{\text { Abstract }}$
This study presents a semi-analytical solution method to analyze the geometrically nonlinear response of bonded composite single-lap joints with tapered adherend edges under uniaxial tension. The solution method provides the transverse shear and normal stresses in the adhesive and in-plane stress resultants and bending moments in the adherends. The method utilizes the principle of virtual work in conjunction with von Karman's nonlinear plate theory to model the adherends and the shear lag model to represent the kinematics of the thin adhesive layer between the adherends. Furthermore, the method accounts for the bilinear elastic material behavior of the adhesive while maintaining a linear stress-strain relationship in the adherends. In order to account for the stiffness changes due to thickness variation of the adherends along the tapered edges, their in-plane and bending stiffness matrices are varied as a function of thickness along the tapered region. The combination of these complexities results in a system of nonlinear governing equilibrium equations. This approach represents a computationally efficient alternative to finite element method. Comparisons are made with corresponding results obtained from finite-element analysis. The results confirm the validity of the solution method. The numerical results present the effects of taper angle, adherend overlap length, and the bilinear adhesive material on the stress fields in the adherends, as well as the adhesive, of a single-lap joint.
\end{abstract}

*Graduate Research Assistant, Department of Aerospace and Mechanical Engineering.

${ }^{\dagger}$ Assistant Research Professor, Department of Aerospace and Mechanical Engineering.

$\$$ Professor, Department of Aerospace and Mechanical Engineering. Member AIAA.

${ }^{\S}$ Aerospace Research Engineer, Mechanics and Durability Branch, Senior Member AIAA.

"Head, Mechanics and Durability Branch. Associate Fellow AIAA.

Copyright (C) 2004 by the American Institute of Aeronautics and Astronautics. All rights reserved.

\section{Nomenclature}

$A^{(p)} \quad=$ area of adherends and adhesive

$\Gamma^{(p)} \quad=$ boundary of adherends and adhesive

$\Gamma_{m}^{(p)} \quad=m^{\text {th }}$ boundary segment

$\mathbf{n}_{m}^{(p)} \quad=$ unit normal to the $m^{\text {th }}$ boundary segment

$z^{(p)} \quad=$ reference planes

$h^{(p)} \quad=$ half thickness of adhesive and adherends

$\zeta^{(p)}=$ coordinates on reference planes

$t_{k}^{(p)} \quad=$ ply thickness

$\theta_{k}^{(p)} \quad=$ ply orientation

$E_{L}^{(p)}, E_{T}^{(p)}, G_{L T}^{(p)}, v_{L T}^{(p)}=$ ply material properties

$\bar{Q}_{i j(k)}^{(p)}=$ coefficients of reduced ply stiffness matrix

$\mathbf{A}^{(p)}, \mathbf{D}^{(p)}, \mathbf{B}^{(p)}=$ in-plane, bending, and coupled stiffness matrices

$E_{\text {eff }}^{(a)} \quad=$ effective adhesive Young's modulus

$G_{\text {eff }}^{(a)} \quad=$ effective adhesive shear modulus

$v^{(a)} \quad=$ adhesive Poisson's ratio

$\tau_{e f f}^{(a)}, \gamma_{e f f}^{(a)}=$ effective transverse shear stress and shear strains

$K=$ degree of the B-spline functions

$\mathbf{t}_{\beta}^{(p)} \quad=$ pre-selected knot points of B-spline functions

$T_{m}\left(x ; \mathbf{t}_{x}^{(p)}, K\right), T_{n}\left(y ; \mathbf{t}_{y}^{(p)}, K\right)=\mathrm{B}$-spline functions

$M_{\beta}^{(p)}=$ extent of of B-spline functions

$c_{\alpha(m n)}^{(p)}=$ unknown coefficients in B-spline functions

$\mathbf{c}_{\alpha}^{(p)}=$ unknown vector of coefficients, $c_{\alpha m n}^{(p)}$

$t_{x}, t_{y}, t_{z}=$ external in-plane tractions

$m_{x}, m_{y}=$ external bending moments

$\mathbf{N}^{(p)}, \mathbf{M}^{(p)}=$ in-plane stress resultants and bending moments

$U_{x}^{(p)}, U_{y}^{(p)}, U_{z}^{(p)}=$ global displacements

$\hat{\mathbf{u}}_{m}^{(p)} \quad=$ prescribed displacements and slopes

$\mathbf{q}^{(p)} \quad=$ unknowns in governing equations

$e_{\alpha \beta}^{(p)} \quad=$ strain components

$\mathbf{e}^{(p)} \quad=$ total strain vector

$\boldsymbol{\kappa}_{x x}^{(p)}, \boldsymbol{\kappa}_{y y}^{(p)}, \boldsymbol{\kappa}_{x y}^{(p)}=$ bending strain (curvature) resultants 
$\varepsilon_{x x}^{(p)}, \varepsilon_{y y}^{(p)}, \gamma_{x y}^{(p)}=$ in-plane strain resultants

$\varepsilon^{(p)}, \boldsymbol{\kappa}^{(p)}=$ in-plane strain resultant and curvature vectors

$\sigma_{z z}^{(a)}, \varepsilon_{z z}^{(a)}=$ adhesive normal stress and strain

$\sigma_{x z}^{(a)}, \sigma_{y z}^{(a)}=$ adhesive transverse shear stresses

$\gamma_{\alpha z}^{(a)}, \varepsilon_{z z}^{(a)}=$ adhesive transverse shear and normal strains

$\boldsymbol{\varepsilon}^{(a)}=$ transverse shear and normal strains in adhesive

$\Lambda_{\alpha m}^{(p)}, \Lambda_{z m}^{\prime(p)}=$ Lagrange multiplier functions

$\Lambda_{m}^{(p)} \quad=$ matrix of Lagrange multiplier functions

$\delta W_{i}, \delta W_{e}=$ virtual work of internal and external forces

$\delta W_{i}^{(p)} \quad=$ internal virtual work of adherends and adhesive

$\delta W_{c}^{(p)}=$ virtual work of boundary reaction forces

$V_{c} \quad=$ potential energy of the constraint forces

\section{Introduction}

The reduction of the transverse shear and normal stress concentrations along the edges of adhesive bondlines is important in order to prevent premature failure of the bonded joint. The determination of the complete stress and strain fields in composite bonded single-lap joints presents difficulties arising from the step-wise geometry, material property variations, laminated construction of the adherends, the bilinear material behavior of the adhesive, as well as the effect of tension induced stiffening (geometrically nonlinear effect) on the bending deformation of the adherends subjected to uniaxial tension. Also, the local stress variations near the ends of the overlap region are characterized by very high gradients or even analytically predicted singularities. The sharp gradients of the stress components depend on the elastic properties of the adherends and adhesive as well as the joint geometry. These peak transverse normal and shear stresses in the adhesive can be reduced by tapering the adherends toward the ends.

In order to facilitate the use of lap joints in present and future structures, the analysis of the geometrically nonlinear response of bonded single-lap joints has received considerable attention over the past two decades. Previous analyses of bonded lap joints can be categorized as "shear-lag" and "finite-element" models. An extensive review and in-depth discussion of the previous investigations can be found in articles by Tsai and Morton, ${ }^{1}$ Ding and Kumosa, ${ }^{2}$ and Osnes and Andersen. ${ }^{3}$ Due to the aforementioned complexities, the majority of the investigations have utilized the finite element method in determining the stress and the strain field in a bonded lap joint. However, in many of these investigations, the three-dimensional description of the bonded lap joint was simplified to a two-dimensional analysis under certain assumptions. ${ }^{4-7}$

In order to enhance computational efficiency, Penado $^{8}$ introduced an approach based on the substructuring technique. In this approach, the general response of the bonded lap joint is obtained analytically from the solution of force-moment equilibrium conditions. The analytically evaluated force-moment values at the overlap ends are then used as the natural boundary condition for a highly detailed twodimensional finite element analysis of the overlap joint under the assumption that the overlap ends are simply supported.

Combining the shear-lag model of Goland and Reissner ${ }^{9}$ with a detailed finite element modeling of the adherends with three-dimensional elements, Edlund and Klarbring ${ }^{10}$ employed the principle of virtual work to analyze the geometrically nonlinear response of bonded single-lap joints with a linearly elastic adhesive. The shear-lag model approximates the transverse shear and normal strain components in terms of the relative displacements of the adherends. An alternative to the shear-lag model is to model the adhesive with one or two layers of brick elements and assemble these elements with the brick elements of the adherends. However, as mentioned before, the size of the brick elements used in the adhesive might introduce aspect ratio problems. The recent investigations by Pandey and Narasimhan ${ }^{11}$ and Narasimhan and Pandey ${ }^{12}$ utilized this approach for solving the three-dimensional large deflection analysis of single-lap joints with viscoelastic adhesive behavior.

In the finite element analysis, the adhesive requires a highly refined mesh in order to keep the proper aspect ratio between the elements in the adherends and adhesive. Therefore, the major advantage of the twodimensional finite element models over the threedimensional models is the significant reduction of the number of degrees of freedom.

Furthermore, for an incremental-iterative solution of the governing equations in which the global stiffness matrix is repeatedly calculated, the three-dimensional finite element analysis of the entire domain becomes computationally demanding.

Thus, it is beneficial to have an efficient specialpurpose analysis method that can be used to conduct extensive parametric studies in a timely manner and at relatively low computational costs. However, there is no analytical or semi-analytical approach for determining the three-dimensional response of the geometrically nonlinear analysis of bonded single-lap joints. Therefore, the goal of the present study is to develop a three-dimensional analysis method that is well suited for parametric studies that accurately 
predicts the geometrically nonlinear behavior of a bonded single-lap, tapered, composite joint subjected to uniaxial tension. In particular, this study focuses on the effects of geometric nonlinearity, tapering of the adherend thickness, changes in the overlap length of the adherends, and the linear and bilinear elastic behaviors of the adhesive on the in-plane stresses in the adherends and the transverse normal and transverse shear stresses in the adhesive of the joint.

In the remainder of this paper, details of the analysis method are presented and results from this approach are discussed. First, the boundary-value problem is defined. Next, the analysis details and numerical solution procedure are described. Then, results for two bonded lap-joint configurations are presented.

\section{Problem Statement}

The bonded single-lap joint configuration consists of two rectangular composite adherends bonded through a thin layer of adhesive, as shown in Fig. 1. While the adhesive has a uniform thickness, the composite adherends have tapered edges. The tapered edges of the adherends are used to reduce stress concentrations along theedges. Both the geometry and kinematics of the adherends and adhesive are described by utilizing the global coordinate system $(x, y, z)$, as shown in Fig. 1 .

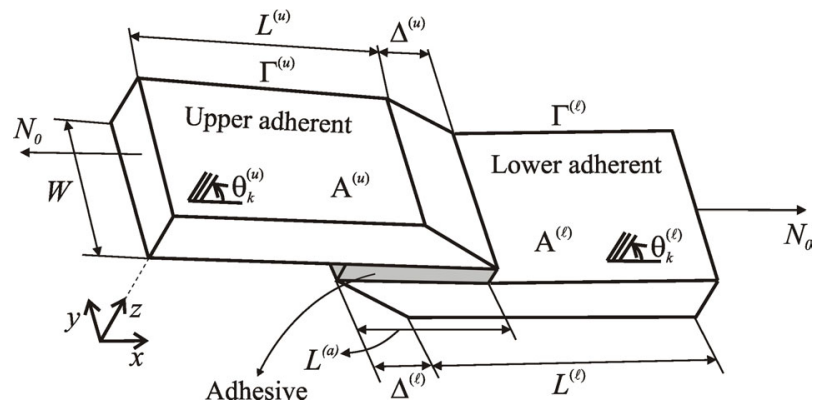

Fig. 1 Description of geometry, fiber angle, and loading of a bonded single-lap joint subjected to uniaxial loading.

The lower and upper adherends are identified by the super scripts $u$ and $\ell$, and their associated area and boundaries are denoted by $A^{(u)}$ and $A^{(\ell)}$ and $\Gamma^{(u)}$ and $\Gamma^{(\ell)}$, respectively. The adhesive is identified by the subscript $a$ and its associated area and boundaries are represented by $A^{(a)}$ and $\Gamma^{(a)}$, respectively. As shown in Fig. 1, both the adherends and adhesive have a rectangular boundary geometry. Hence, their boundaries can be represented by dividing their entire boundaries into four straight boundary segments, i.e,

$$
\Gamma^{(p)}=\sum_{m=1}^{4} \Gamma_{m}^{(p)} \quad(p=u, \ell, a)
$$

in which $\Gamma_{m}^{(p)}$ denotes the $m^{\text {th }}$ boundary segment around the adherend and adhesive boundary. Along the adherend and adhesive boundaries, $\Gamma_{p} \quad(p=u, \ell, a)$, the unit normal to the $m^{\text {th }}$ boundary segment is represented by $\mathbf{n}_{m}^{(p)}$, with components $n_{x m}^{(p)}$ and $n_{y m}^{(p)}$ in the $x$ - and $y$-directions, respectively. The unit normal, $\mathbf{n}_{m}^{(p)}$, makes an angle, $\phi_{m}^{(p)}$, with respect to the positive $x$-axis, as shown in Fig. 1 .

The exterior edges of the adherends are subjected to both in-plane tractions and bending moments. The inplane external tractions include components $t_{x}, t_{y}$ and $t_{z}$, and the external bending tractions include components $m_{x}$ and $m_{y}$. The traction components are defined with respect to the $(x, y, z)$ structural coordinates, and their positive-valued directions are shown in Fig. 1. In the adherends and adhesive, the global displacement components in the $x$-, $y$-, and $z$-directions are denoted by $U_{x}^{(p)}, U_{y}^{(p)}$, and $U_{z}^{(p)}$, with $p=u, \ell, a$, respectively.

The laminated adherends are made of specially orthotropic layers. Each layer has a thickness of $t_{k}^{(p)}$ and orientation angle of $\theta_{k}^{(p)}(p=u, \ell)$, which is defined with respect to the positive $x$-axis, as shown in Fig. 1. Also, the orthotropic material properties of each layer include the elastic moduli $E_{L}^{(p)}$ and $E_{T}^{(p)}$, shear modulus $G_{L T}^{(p)}$, and Poisson's ratio $v_{L T}^{(p)}$, where $L$ and $T$ are the longitudinal and transverse directions, respectively.

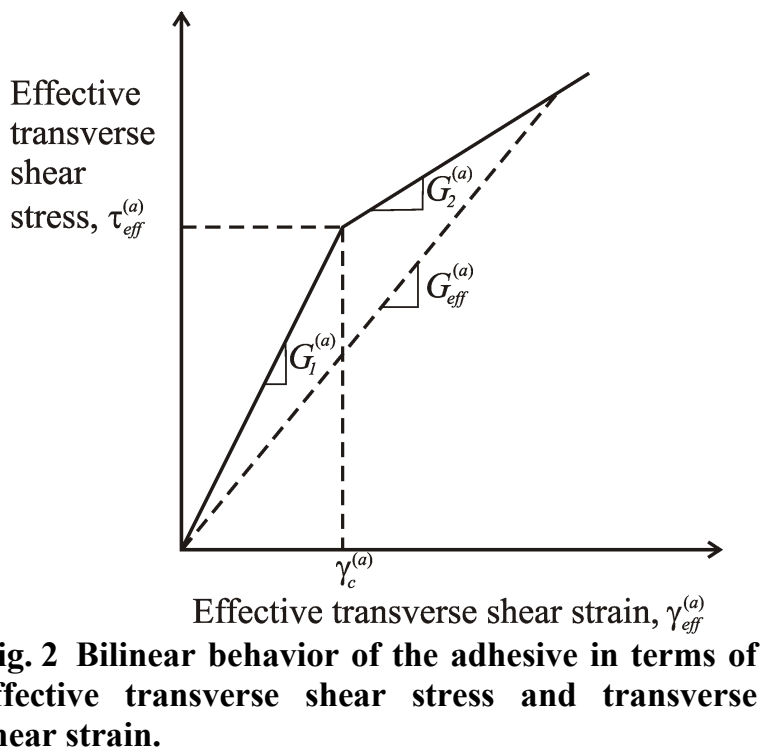


The adhesive material is isotropic, homogeneous, and elastic with a bilinear relation between the effective transverse shear stress, $\tau_{\text {eff }}^{(a)}$, and effective transverse shear strain, $\gamma_{\text {eff }}^{(a)}$, as shown in Fig. 2. The effective transverse shear stress and strain are defined by

$$
\begin{gathered}
\tau_{e f f}^{(a)}=\sqrt{\sigma_{x z}^{(a)^{2}}+\sigma_{y z}^{(a)^{2}}} \\
\gamma_{e f f}^{(a)}=\sqrt{\gamma_{x z}^{(a)^{2}}+\gamma_{y z}^{(a)^{2}}}
\end{gathered}
$$

in which $\sigma_{x z}^{(a)}$ and $\sigma_{y z}^{(a)}$ represent the components of the transverse shear stress and $\gamma_{x z}^{(a)}$ and $\gamma_{y z}^{(a)}$ represent the components of the transverse shear strain in the adhesive. As shown in Fig. 2, the initial shear modulus of the bilinear adhesive behavior is denoted by $G_{1}^{(a)}$, and it reduces to $G_{2}^{(a)}$ after the characteristic transverse shear strain, $\gamma_{c}^{(a)}$. It has a Poisson's ratio of $v^{(a)}$. With these parameters, the bilinear relationship between the effective transverse shear stress, $\tau_{\text {eff }}^{(a)}$, and effective transverse shear strain, $\gamma_{\text {eff }}^{(a)}$, can be expressed as

$$
\begin{aligned}
\tau_{e f f}^{(a)} & =G_{1}^{(a)} \gamma_{e f f}^{(a)}\left[1-H\left(\gamma_{e f f}^{(a)}-\gamma_{c}^{(a)}\right)\right] \\
& +\left[G_{1}^{(a)} \gamma_{c}^{(a)}+G_{2}^{(a)}\left(\gamma_{e f f}^{(a)}-\gamma_{c}^{(a)}\right)\right] \times H\left(\gamma_{e f f}^{(a)}-\gamma_{c}^{(a)}\right)
\end{aligned}
$$

where $H\left(\gamma_{\text {eff }}^{(a)}-\gamma_{c}^{(a)}\right)$ is the Heaviside step function.

In accordance with this relationship, the transverse shear stresses, $\sigma_{\alpha z}^{(a)}$, and strains, $\gamma_{\alpha z}^{(a)}$, are related by

$$
\sigma_{\alpha z}^{(a)}=G_{e f f}^{(a)} \gamma_{\alpha z}^{(a)} \quad(\alpha=x, y)
$$

in which the parameters $G_{\text {eff }}^{(a)}$ represent the effective shear modulus of the adhesive, defined as

$$
G_{e f f}^{(a)}=\frac{\tau_{e f f}^{(a)}}{\gamma_{e f f}^{(a)}}
$$

Furthermore, the transverse normal stress, $\sigma_{z z}^{(a)}$, and strain, $\varepsilon_{z z}^{(a)}$, in the adhesive are related by

$$
\sigma_{z z}^{(a)}=E_{e f f}^{(a)} \varepsilon_{z z}^{(a)}
$$

in which $E_{\text {eff }}^{(a)}$ is the effective Young's modulus expressed as

$$
E_{\text {eff }}^{(a)}=2 G_{\text {eff }}^{(a)}\left(1+v^{(a)}\right)
$$

While the thickness of the adhesive is uniform and denoted by $2 h^{(a)}$, the adherends have variable thicknesses, $h^{(p)}(x, y)$, with $p=u, \ell$, due to their tapered shape near the edges. The thicknesses of the adherends are defined as discrete linear functions of the in-plane coordinates. The reference planes of the adherends and adhesive, denoted by $z^{(p)}$, with $p=u, \ell, a$, are shown in Fig. 3 .

The problem posed here concerns the development of a three-dimensional semi-analytical method to determine the displacement and stress fields in bonded single-lap joints while including the effects of geometric nonlinearity and bilinear elastic adhesive material behavior. The capability of the method is demonstrated by considering first a quasi-isotropic tapered adherends and a bilinear adhesive material behavior. The second configuration concerns the effect of the overlap length of the adherends on the general response of both the adherends and adhesive. The lap joints are simply supported along the left and right edges of the upper and lower adherends, respectively. The left edge of the upper adherend is also restrained against any horizontal movement. Furthermore, the mid points of the left and right edges of the lower and upper adherends are restrained to move in the $y$ direction so that the rigid-body movement of the lap joint is completely suppressed.

\section{Solution Method}

The present three-dimensional geometrically nonlinear analysis method is based on the principle of virtual work. The displacement components are approximated in terms of the B-spline functions ${ }^{13}$ in a double series representation as

$u_{\alpha}^{(p)}=\sum_{m=0}^{M_{x}^{(p)}} \sum_{n=0}^{M_{y}^{(p)}} c_{\alpha(m n)}^{(p)} T_{m}\left(x ; \mathbf{t}_{x}^{(p)}, K\right) T_{n}\left(y ; \mathbf{t}_{y}^{(p)}, K\right)$

in which $c_{\alpha(m n)}^{(p)}$, with $p=u, \ell$ and $\alpha=x, y, z$, are the unknown coefficients. The parameter $M_{\beta}^{(p)}$, with $\beta=x, y$ and $p=u, l$, specifies the extent of the series and the knot vector $\mathbf{t}_{\beta}^{(p)}$ contains pre-selected knot points (coordinates) in the direction of $\beta$. These knot points are used to increase the accuracy of the B-splines at certain locations. Also, the parameter $K$ controls the degree of the B-spline functions and, consequently, the continuity of the field variable. The $K^{\text {th }}$-order B-spline function is comprised of the $(K-1)^{\text {th }}$-order of polynomials. 


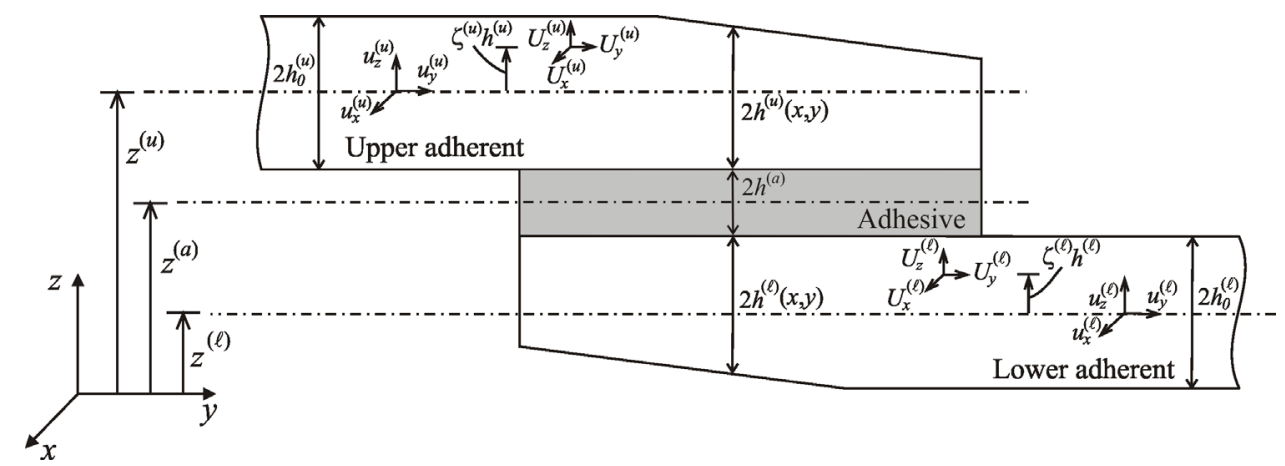

Fig. 3 The reference surface and kinematics of the bonded single-lap joint.

The details of the B-spline functions along with the definitions of the parameters $\mathbf{t}_{\beta}^{(p)}$ and $K$ are given in the Appendix .These displacement functions can be expressed in matrix form as

$$
\begin{aligned}
& u_{x}^{(p)}=\mathbf{V}^{(p)^{\mathrm{T}}} \mathbf{c}_{x}^{(p)} \\
& u_{y}^{(p)}=\mathbf{V}^{(p)^{\mathrm{T}}} \mathbf{c}_{y}^{(p)} \\
& u_{z}^{(p)}=\mathbf{V}^{(p)^{\mathrm{T}}} \mathbf{c}_{z}^{(p)}
\end{aligned}
$$

in which the vectors $\mathbf{c}_{\alpha}^{(p)}$, with $\alpha=x, y, z$, contain the unknown coefficients (generalized coordinates) $c_{\alpha m n}^{(p)}$. The known vectors, $\mathbf{V}^{(p)}$, are expressed in terms of the B-spline functions $T_{m}\left(x ; \mathbf{t}_{x}^{(p)}, K\right)$ and $T_{n}\left(y ; \mathbf{t}_{y}^{(p)}, K\right)$. In matrix form, the approximate displacement representations of Eqs. (9a-c) are rewritten as

$$
\begin{aligned}
& u_{x}^{(p)}=\mathbf{V}_{x}^{(p)^{\mathrm{T}}} \mathbf{q}^{(p)} \\
& u_{y}^{(p)}=\mathbf{V}_{y}^{(p)^{\mathrm{T}}} \mathbf{q}^{(p)} \\
& u_{z}^{(p)}=\mathbf{V}_{z}^{(p)^{\mathrm{T}}} \mathbf{q}^{(p)}
\end{aligned}
$$

in which the known vectors, $\mathbf{V}_{\alpha}^{(p)}$, with $\alpha=x, y, z$, are defined as

$$
\begin{aligned}
\mathbf{V}_{x}^{(p)^{\mathrm{T}}} & =\left\{\mathbf{V}^{(p)}, \mathbf{0}, \mathbf{0}\right\} \\
\mathbf{V}_{y}^{(p)^{\mathrm{T}}} & =\left\{\mathbf{0}, \mathbf{V}^{(p)}, \mathbf{0}\right\} \\
\mathbf{V}_{z}^{(p)^{\mathrm{T}}} & =\left\{\mathbf{0}, \mathbf{0}, \mathbf{V}^{(p)}\right\}
\end{aligned}
$$

The unknown vector $\mathbf{q}^{(p)}$ is defined as

$$
\mathbf{q}^{(p)^{\mathrm{T}}}=\left\{\mathbf{c}_{x}^{(p)^{\mathrm{T}}}, \mathbf{c}_{y}^{(p)^{\mathrm{T}}}, \mathbf{c}_{z}^{(p)^{\mathrm{T}}}\right\}
$$

Note that the series representation of the displacement components is not required to satisfy any type of kinematic admissibility.

\section{Displacement Components}

The adherends interacting through the adhesive, which sustains transverse normal and shear deformations but not in-plane deformation, are subjected to in-plane and bending deformations but not transverse normal and shear deformations. The transverse normal and shear strain components in the adherends are disregarded because they are thin. Therefore, the in-plane strain components in the adhesive, and the transverse normal and shear strain components in the adherends, are not included in the derivation of the kinematic relations.

In accordance with the Kirchhoff plate theory, the global displacement components, $U_{x}^{(p)}, U_{y}^{(p)}$, and $U_{z}^{(p)}$ in each of the adherends are defined as

$$
\begin{aligned}
& U_{\alpha}^{(p)}(x, y, z)=u_{\alpha}^{(p)}(x, y)-\zeta^{(p)} h^{(p)} u_{z, \alpha}^{(p)} \\
& U_{z}^{(p)}(x, y, z)=u_{z}^{(p)}(x, y)
\end{aligned}
$$

for which $p=u, \ell$ and $\alpha=x, y$, and the displacement components, $U_{x}^{(p)}, U_{y}^{(p)}$, and $u_{z}^{(p)}$, are defined on the reference surfaces with respect to the global Cartesian coordinates $\left(x^{(p)}, y^{(p)}, z^{(p)}\right)$, as shown in Fig. 3. In Eq. (14), the subscript after a comma indicates differentiation with respect to the variable. The coordinate $\zeta^{(p)}$ located on each of the reference planes is defined as

$$
\zeta^{(p)}=\frac{z-z^{(p)}}{h^{(p)}} \quad(p=u, \ell)
$$


and varies in the range $-1 \leq \zeta^{(p)} \leq 1$, with $p=u$, $\ell$. The thicknesses of the adherends are specified by $2 h^{(p)}(x, y)$, in which $h^{(p)}(x, y)=h_{0}^{(p)}$ in the untapered sections of the adherends. Also, the location of the reference planes with respect to the global coordinate system $(x, y, z)$ are defined by $z^{(p)}$, with $p=u, \ell$, which are located at the mid-surfaces (with respect to the untapered thickness) of the adherends, as shown in Fig. 3.

\section{Strain-Displacement Relations}

The strain measure for the adherends is based on the modified form of Green's nonlinear strain displacement relations in conjunction with von Karman assumptions for large deformation of plates ${ }^{14}$. Therefore, the strain components in the adherends, $e_{\alpha \beta}^{(p)}(p=u, \ell ; \alpha, \beta=$ $x, y)$, can be expressed as

$$
\begin{aligned}
& e_{x x}^{(p)}=\varepsilon_{x x}^{(p)}-\zeta^{(p)} h^{(p)} \kappa_{x x}^{(p)}+\frac{1}{2}\left(u_{z, x}^{(p)}\right)^{2} \\
& e_{y y}^{(p)}=\varepsilon_{y y}^{(p)}-\zeta^{(p)} h^{(p)} \kappa_{y y}^{(p)}+\frac{1}{2}\left(u_{z, y}^{(p)}\right)^{2} \\
& e_{x y}^{(p)}=\frac{1}{2} \gamma_{x y}^{(p)}+\frac{1}{2} \zeta^{(p)} h^{(p)} \kappa_{x y}^{(p)}+\frac{1}{2} u_{z, x}^{(p)} u_{z, y}^{(p)}
\end{aligned}
$$

in which

$$
\begin{aligned}
& \varepsilon_{x x}^{(p)}=u_{x, x}^{(p)} \quad ; \quad \kappa_{x x}^{(p)}=-u_{z, x x}^{(p)} \\
& \varepsilon_{y y}^{(p)}=u_{y, y}^{(p)} \quad ; \quad \kappa_{y y}^{(p)}=-u_{z, y y}^{(p)} \\
& \gamma_{x y}^{(p)}=u_{x, y}^{(p)}+u_{y, x}^{(p)} ; \quad \kappa_{x y}^{(p)}=-2 u_{z, x y}^{(p)}
\end{aligned}
$$

where $\varepsilon_{x x}^{(p)}, \varepsilon_{y y}^{(p)}$, and $\gamma_{x y}^{(p)}$ represent the in-plane strain resultants and $\boldsymbol{\kappa}_{x x}^{(p)}, \boldsymbol{\kappa}_{y y}^{(p)}$, and $\boldsymbol{\kappa}_{x y}^{(p)}$ represent the bending strain (curvature) resultants on the reference surfaces. Also, the in-plane and bending (curvature) strain resultants constitute the components of the linear part of the in-plane strain, $\varepsilon_{L}^{(p)}$, and curvature, $\boldsymbol{\kappa}_{L}^{(p)}$, vectors in the form

$$
\begin{aligned}
& \boldsymbol{\varepsilon}_{L}^{(p)^{\mathrm{T}}}=\left\{\varepsilon_{x x}^{(p)}, \varepsilon_{y y}^{(p)}, \gamma_{x y}^{(p)}\right\}=\left\{u_{x, x}^{(p)}, u_{y, y}^{(p)}, u_{x, y}^{(p)}+u_{y, x}^{(p)}\right\} \\
& \boldsymbol{\kappa}_{L}^{(p)^{\mathrm{T}}}=\left\{\boldsymbol{\kappa}_{x x}^{(p)}, \boldsymbol{\kappa}_{y y}^{(p)}, \boldsymbol{\kappa}_{x y}^{(p)}\right\}=\left\{-u_{z, x x}^{(p)},-u_{z, y y}^{(p)},-2 u_{z, x y}^{(p)}\right\}
\end{aligned}
$$

Similarly, the nonlinear terms appearing in the strain components, $e_{\alpha \beta}^{(p)}(\alpha, \beta=x, y)$, are included in the nonlinear part of the in-plane strain resultant vector, $\varepsilon_{N}^{(p)}$, in the form

$$
\boldsymbol{\varepsilon}_{N}^{(p)^{\mathrm{T}}}=\left\{\frac{1}{2}\left(u_{z, x}^{(p)}\right)^{2}, \frac{1}{2}\left(u_{z, y}^{(p)}\right)^{2}, u_{z, x}^{(p)} u_{z, y}^{(p)}\right\}
$$

Although the bending deformations (curvatures) are only linearly related to the out-of-plane displacement component, $u_{z}^{(p)}$, for consistency, a zero-valued vector is employed to represent the nonlinear part of the curvature vector, $\boldsymbol{\kappa}_{N}^{(p)}$, as

$$
\boldsymbol{\kappa}_{N}^{(p)}=\{0,0,0\}
$$

Substituting for the derivatives of the displacement components from Eq. (10), the linear and nonlinear parts of the in-plane strain resultant and curvature vectors can be expressed as

$$
\begin{aligned}
& \boldsymbol{\varepsilon}_{L}^{(p)}=\mathbf{L}_{\varepsilon_{L}}^{(p)} \mathbf{q}^{(p)}, \boldsymbol{\kappa}_{L}^{(p)}=\mathbf{L}_{\kappa_{L}}^{(p)} \mathbf{q}^{(p)} \\
& \boldsymbol{\varepsilon}_{N}^{(p)}=\mathbf{L}_{\varepsilon_{N}}^{(p)}\left(\mathbf{q}^{(p)}\right) \mathbf{q}^{(p)}, \boldsymbol{\kappa}_{N}^{(p)}=0
\end{aligned}
$$

where

$$
\begin{aligned}
& \mathbf{L}_{\varepsilon_{L}}^{(p)}=\left[\begin{array}{c}
\mathbf{V}_{x, x}^{(p)^{\mathrm{T}}} \\
\mathbf{V}_{y, y}^{(p)^{\mathrm{T}}} \\
\mathbf{V}_{x, y}^{(p)^{\mathrm{T}}}+\mathbf{V}_{y, x}^{(p)^{\mathrm{T}}}
\end{array}\right] \\
& \mathbf{L}_{\kappa_{L}}^{(p)}=-\left[\begin{array}{c}
\mathbf{V}_{z, x x}^{(p)^{\mathrm{T}}} \\
\mathbf{V}_{z, y)^{\mathrm{T}}}^{(p)} \\
2 \mathbf{V}_{z, x y}^{(p)^{\mathrm{T}}}
\end{array}\right] \\
& \mathbf{L}_{\varepsilon_{N}}^{(p)}\left(\mathbf{q}^{(p)}\right)=\frac{1}{2}\left[\begin{array}{c}
u_{z, x}^{(p)} \mathbf{V}_{z, x}^{(p)^{\mathrm{T}}} \\
u_{z, y}^{(p)} \mathbf{V}_{z, y}^{(p)^{\mathrm{T}}} \\
u_{z, x}^{(p)} \mathbf{V}_{z, y}^{(p)^{\mathrm{T}}}+u_{z, y}^{(p)} \mathbf{V}_{z, x}^{(p)^{\mathrm{T}}}
\end{array}\right]
\end{aligned}
$$

The vectors of strain resultants defined in Eq. (20) can be combined in a compact form as

$$
\mathbf{e}_{\alpha}^{(p)}=\mathbf{L}_{\alpha}^{(p)} \mathbf{q}^{(p)} \quad(\alpha=L, N)
$$

where

$$
\mathbf{e}_{\alpha}^{(p)^{\mathrm{T}}}=\left\{\boldsymbol{\varepsilon}_{\alpha}^{(p)^{\mathrm{T}}}, \boldsymbol{\kappa}_{\alpha}^{(p)^{\mathrm{T}}}\right\}, \quad \mathbf{L}_{L}^{(p)}=\left[\begin{array}{c}
\mathbf{L}_{\varepsilon_{L}}^{(p)} \\
\mathbf{L}_{\kappa_{L}}^{(p)}
\end{array}\right]
$$




$$
\mathbf{L}_{N}^{(p)}\left(\mathbf{q}^{(p)}\right)=\left[\begin{array}{c}
\mathbf{L}_{\varepsilon_{N}}^{(p)}\left(\mathbf{q}^{(p)}\right) \\
0
\end{array}\right]
$$

Furthermore, the linear and nonlinear parts of the strain vectors, $\mathbf{e}_{L}^{(p)}$ and $\mathbf{e}_{N}^{(p)}$, can be added to form the total strain vector as

$$
\begin{aligned}
\mathbf{e}^{(p)} & =\mathbf{e}_{L}^{(p)}+\mathbf{e}_{N}^{(p)}=\left[\mathbf{L}_{L}^{(p)}+\mathbf{L}_{N}^{(p)}\left(\mathbf{q}^{(p)}\right)\right] \mathbf{q}^{(p)} \\
& =\mathbf{H}^{(p)}\left(\mathbf{q}^{(p)}\right) \mathbf{q}^{(p)}
\end{aligned}
$$

where

$$
\mathbf{H}^{(p)}\left(u_{z}^{(p)}\right)=\mathbf{L}_{L}^{(p)}+\mathbf{L}_{N}^{(p)}\left(\mathbf{q}^{(p)}\right)
$$

For the adhesive bonding of the two adherends, the displacement components are assumed to vary linearly through the thickness ${ }^{1}$. Although the adhesive undergoes the same magnitudes of the in-plane and transverse displacements as those of the adherends, the strain measure is based on a linear shear-lag model, where the transverse shear strain and the normal strain components in the adhesive are expressed as

$$
\begin{aligned}
\gamma_{\alpha z}^{(a)} & =\frac{1}{2 h^{(a)}}\left[U_{\alpha}^{(u)}(x, y, 0)-U_{\alpha}^{(\ell)}(x, y, 0)\right] \\
& +\frac{1}{2}\left[\left(1+\zeta^{(a)}\right) U_{z, \alpha}^{(u)}(x, y, 0) \quad \alpha=x, y\right. \\
& \left.+\left(1-\zeta^{(a)}\right) U_{z, \alpha}^{(\ell)}(x, y, 0)\right] \\
\varepsilon_{z z}^{(a)} & =\frac{1}{2 h^{(a)}}\left[u_{z}^{(u)}(x, y)-u_{z}^{(\ell)}(x, y)\right]
\end{aligned}
$$

Finally, substituting from Eq. (10) for the displacement components in Eq. (26) leads to the strain vector containing the transverse shear and normal strain components in the adhesive as

$$
\boldsymbol{\varepsilon}^{(a)}=\mathbf{L}_{a}^{(u)} \mathbf{q}^{(u)}-\mathbf{L}_{a}^{(\ell)} \mathbf{q}^{(\ell)}
$$

where

$$
\boldsymbol{\varepsilon}^{(a) T}=\left\{\gamma_{x z}^{(a)}, \gamma_{y z}^{(a)}, \varepsilon_{z z}^{(a)}\right\}
$$

and the matrices $\mathbf{L}_{a}^{(p)}$ are defined as

$$
\begin{aligned}
& \mathbf{L}_{a}^{(u)}=\mathbf{L}_{a \varepsilon}^{(u)}+\mathbf{L}_{a \kappa}^{(u)} \\
& \mathbf{L}_{a}^{(\ell)}=\mathbf{L}_{a \varepsilon}^{(\ell)}-\mathbf{L}_{a \kappa}^{(\ell)}
\end{aligned}
$$

in which

$$
\begin{gathered}
\mathbf{L}_{a \varepsilon}^{(p)}=\frac{1}{2 h^{(a)}}\left[\begin{array}{c}
\mathbf{V}_{x}^{(p)^{\mathrm{T}}} \\
\mathbf{V}_{y}^{(p)^{\mathrm{T}}} \\
\mathbf{0}^{T}
\end{array}\right] \quad(p=\ell, u) \\
\mathbf{L}_{a \kappa}^{(p)}=\frac{1}{2 h^{(a)}}\left[\begin{array}{c}
h^{(p)} \mathbf{V}_{z, x}^{(p)^{T}} \\
h^{(p)} \mathbf{V}_{z, y}^{(p)^{T}} \\
(-1)^{\delta_{p \ell}} \mathbf{V}_{z}^{(p)^{T}}
\end{array}\right](p=\ell, u)
\end{gathered}
$$

\section{Stress-Strain Relations}

The external uniaxial in-plane loads acting along the boundary of the adherends, as shown in Fig. 1, result in not only in-plane stresses but also in bending moments in the adherends due to the couple formed by the eccentric location of the applied in-plane forces. The resulting transverse normal (peeling) stresses created in the adhesive are also due to this couple, which causes the bending deformations in the adherends.

The in-plane stress resultants and bending moments generated by the applied external loading in the adherends are related to the in-plane strain resultants and curvatures, which are defined on the mid-surfaces of the upper and lower adherends through the constitutive relation as

$$
\left\{\begin{array}{c}
\mathbf{N}^{(p)} \\
\mathbf{M}^{(p)}
\end{array}\right\}=\left[\begin{array}{ll}
\mathbf{A}^{(p)}(x, y) & \mathbf{B}^{(p)}(x, y) \\
\mathbf{B}^{(p)}(x, y) & \mathbf{D}^{(p)}(x, y)
\end{array}\right]\left\{\begin{array}{c}
\varepsilon^{(p)} \\
\boldsymbol{\kappa}^{(p)}
\end{array}\right\}
$$

where

$$
\begin{aligned}
& A_{i j}^{(p)}(x, y)=h^{(p)}(x, y) \sum_{k=1}^{N_{p}}\left(\zeta_{k+1}^{(p)}-\zeta_{k}^{(p)}\right) \bar{Q}_{i j(k)}^{(p)} \\
& B_{i j}^{(p)}(x, y)=\frac{1}{2}\left(h^{(p)}(x, y)\right)^{2} \sum_{k=1}^{N_{p}}\left(\zeta_{k+1}^{(p)^{2}}-\zeta_{k}^{(p)^{2}}\right) \bar{Q}_{i j(k)}^{(p)} \\
& D_{i j}^{(p)}(x, y)=\frac{1}{3}\left(h^{(p)}(x, y)\right)^{3} \sum_{k=1}^{N_{p}}\left(\zeta_{k+1}^{(p)^{3}}-\zeta_{k}^{(p)^{2}}\right) \bar{Q}_{i j(k)}^{(p)}
\end{aligned}
$$

with

$$
\begin{aligned}
& \zeta_{k}^{(\ell)}=\frac{z_{k}(x, y)-z^{(\ell)}}{h^{(\ell)}(x, y)} \\
& \left(k=1, \ldots, N_{\ell} ; z^{(\ell)}-h^{(\ell)} \leq z_{k} \leq z^{(\ell)}+h^{(\ell)}\right) \\
& \zeta_{k}^{(u)}=\frac{z_{k}(x, y)-z^{(u)}}{h^{(u)}(x, y)} \\
& \left(k=1, \ldots, N_{u} ; z^{(u)}-h^{(u)} \leq z_{k} \leq z^{(u)}+h^{(u)}\right)
\end{aligned}
$$


In Eq. (31), the matrices $\mathbf{A}^{(p)}, \mathbf{D}^{(p)}$, and $\mathbf{B}^{(p)}$, with $p=u, \ell$, are associated with in-plane, bending, and coupled in-plane and bending behaviors of the adherends, and $\bar{Q}_{i j(k)}^{(p)}(p=u, \ell)$ are the coefficients of the reduced stiffness matrix of the $k^{\text {th }}$ ply defined in the global $(x-y)$ coordinate system. Note that the tapered adherend thickness, $h^{(p)}(p=u, \ell)$, varies as a function of the $(x-y)$ coordinates. Hence, the material property matrices associated with the adherends, $\mathbf{A}^{(p)}, \mathbf{D}^{(p)}$, and $\mathbf{B}^{(p)}$, are dependent on the in-plane coordinates.

Furthermore, the ratio of the ply thickness to the adherend thickness is assumed to be constant, i.e., $t_{k}^{(p)}(x, y) / h^{(p)}(x, y)=\bar{t}_{k}^{(p)}=$ constant. In this case, the material property matrices, $\mathbf{A}^{(p)}, \mathbf{D}^{(p)}$, and $\mathbf{B}^{(p)}$, become dependent only on the adherend thickness, $h^{(p)}(x, y)$, with $p=u, \ell$.

The relation given in Eq. (31) can be compacted in the form

$$
\mathbf{s}^{(p)}=\mathbf{E}^{(p)} \mathbf{e}^{(p)} \quad(p=u, \ell)
$$

in which $\mathbf{s}^{(p)}, \mathbf{E}^{(p)}$, and $\mathbf{e}^{(p)}$ are defined as

$$
\begin{aligned}
& \mathbf{s}^{(p)^{\mathrm{T}}}=\left\{\mathbf{N}^{(p)^{\mathrm{T}}}, \mathbf{M}^{(p)^{\mathrm{T}}}\right\} \\
& \mathbf{E}^{(p)}=\left[\begin{array}{ll}
\mathbf{A}^{(p)} & \mathbf{B}^{(p)} \\
\mathbf{B}^{(p)} & \mathbf{D}^{(p)}
\end{array}\right] \\
& \mathbf{e}^{(p)^{\mathrm{T}}}=\left\{\boldsymbol{\varepsilon}^{(p)^{\mathrm{T}}}, \boldsymbol{\kappa}^{(p)^{\mathrm{T}}}\right\}
\end{aligned}
$$

With the representation of $\mathbf{e}^{(p)}$ in Eq. (24), the stressstrain relations given in Eq. (35) can be rewritten as

$$
\mathbf{s}^{(p)}=\mathbf{E}^{(p)} \mathbf{H}^{(p)}\left(\mathbf{q}^{(p)}\right) \mathbf{q}^{(p)} \quad(p=u, \ell)
$$

Because the adhesive does not sustain in-plane deformation, the in-plane stress components, $\sigma_{x x}^{(a)}$, $\sigma_{y y}^{(a)}$, and $\sigma_{x y}^{(a)}$, are disregarded. The transverse shear stresses, $\sigma_{x z}^{(a)}$ and $\sigma_{y z}^{(a)}$, and the transverse normal stress, $\sigma_{z z}^{(a)}$, are related to the corresponding strain components through a bilinear relation as

$$
\mathbf{s}^{(a)}=\mathbf{E}^{(a)} \boldsymbol{\varepsilon}^{(a)}
$$

where

$$
\mathbf{s}^{(a)^{\mathrm{T}}}=h^{(a)}\left\{\sigma_{x z}^{(a)}, \sigma_{y z}^{(a)}, \sigma_{z z}^{(a)}\right\}
$$

$$
\begin{aligned}
\boldsymbol{\varepsilon}^{(a) \mathrm{T}^{\mathrm{T}}}=\left\{\gamma_{x z}^{(a)}, \gamma_{y z}^{(a)}, \varepsilon_{z z}^{(a)}\right\} & \mathbf{E}^{(a)}\left(\mathbf{q}^{(s)}, \mathbf{q}^{(p)}\right)=\left[\begin{array}{cc}
G_{e f f}^{(a)}\left(\mathbf{q}^{(s)}, \mathbf{q}^{(p)}\right) & 0 \\
0 & G_{e f f}^{(a)}\left(\mathbf{q}^{(s)}, \mathbf{q}^{(p)}\right) \\
0 & 0 \\
0 & \\
0 & \\
E_{e f f}^{(a)}\left(\mathbf{q}^{(s)}, \mathbf{q}^{(p)}\right)
\end{array}\right]
\end{aligned}
$$

in which the expressions for $G_{e f f}^{(a)}\left(\mathbf{q}^{(s)}, \mathbf{q}^{(p)}\right)=G_{e f f}^{(a)}\left(\gamma_{e f f}^{(a)}\right)$ and $E_{\text {eff }}^{(a)}\left(\mathbf{q}^{(s)}, \mathbf{q}^{(p)}\right)=E_{\text {eff }}^{(a)}\left(\gamma_{\text {eff }}^{(a)}\right)$ are defined in Eqs. (5) and (7), respectively.

Substituting for the expression for $\varepsilon^{(a)}$ from Eq. (27) permits the stress-strain relations given in Eqs. (38) to be expressed in terms of the unknowns of the adherends as

$$
\mathbf{s}^{(a)}=\mathbf{E}^{(a)}\left(\mathbf{q}^{(u)}, \mathbf{q}^{(\ell)}\right)\left(\mathbf{L}_{a}^{(u)} \mathbf{q}^{(u)}-\mathbf{L}_{a}^{(\ell)} \mathbf{q}^{(\ell)}\right)
$$

\section{Boundary Conditions}

Along the $m^{\text {th }}$ segment of the boundary of the adherends, denoted by $\Gamma_{m}^{(p)}$, with $p=u, \ell$, as shown in Fig. 1, the prescribed displacement components normal and tangent to the boundary, ${ }^{(m)} \hat{u}_{n}^{(p)},{ }^{(m)} \hat{u}_{t}^{(p)}$, and ${ }^{(m)} \hat{u}_{z}^{(p)}$, and the slope normal to the boundary, ${ }^{(m)} \hat{u}_{z, n}^{(p)}$, can be imposed as

$u_{n}^{(p)}={ }^{(m)} \hat{u}_{n}^{(p)}$

$u_{t}^{(p)}={ }^{(m)} \hat{u}_{t}^{(p)}$

$u_{z}^{(p)}={ }^{(m)} \hat{u}_{z}^{(p)}$ on $\Gamma_{m}^{(p)} \quad(p=u, \ell ; m=1,2,3,4)$

$u_{z, n}^{(p)}={ }^{(m)} \hat{u}_{z, n}^{(p)}$

Utilizing the vector representations of the displacement components given by Eq. (10), these prescribed displacements can be expressed in vector form as

$$
\mathbf{V}_{m}^{(p)^{\mathrm{T}}} \mathbf{q}^{(p)}-\hat{\mathbf{u}}_{m}^{(p)}=\mathbf{0} \quad(p=u, \ell ; m=1,2,3,4)
$$

where the matrix $\mathbf{V}_{m}^{(p)}$ and the vector $\hat{\mathbf{u}}_{m}^{(p)}$ are defined as 


$$
\begin{aligned}
& \mathbf{V}_{m}^{(p)^{\mathrm{T}}}=\left[\begin{array}{cc}
\cos \phi_{m} \mathbf{V}_{x}^{(p)^{\mathrm{T}}} & \mathbf{0} \\
+\sin \phi_{m} \mathbf{V}_{y}^{(p)^{\mathrm{T}}} & \\
-\sin \phi_{m} \mathbf{V}_{x}^{(p)^{\mathrm{T}}} & \mathbf{0} \\
+\cos \phi_{m} \mathbf{V}_{y}^{(p)^{\mathrm{T}}} & \mathbf{V}_{z}^{(p)^{\mathrm{T}}} \\
\mathbf{0} & \cos \phi_{m} \mathbf{V}_{z, x}^{(p)^{\mathrm{T}}} \\
& +\sin \phi_{m} \mathbf{V}_{z, y}^{(p)}
\end{array}\right] \\
& \hat{\mathbf{u}}_{m}^{(p)^{\mathrm{T}}}=\left\{\begin{array}{llll}
{ }^{(m)} \hat{u}_{n}^{(p)} & { }^{(m)} \hat{u}_{t}^{(p)} & { }^{(m)} \hat{u}_{z}^{(p)} & { }^{(m)} \hat{u}_{z, n}^{(p)}
\end{array}\right\}
\end{aligned}
$$

The boundary conditions in Eq. (41) are enforced as constraint conditions by introducing Lagrange multiplier functions, $\Lambda_{\alpha m}^{(p)}(t)$, with $\alpha=n, t, z$, and $\Lambda_{z m}^{(p)}(t)$, defined along the $m^{t h}$ boundary segment. These boundary conditions are written in integral form as

$$
\begin{array}{r}
\int_{\Gamma_{m}^{(p)}} \Lambda_{m}^{(p)}(t)\left\{\mathbf{V}_{m}^{(p) T} \mathbf{q}^{(p)}-\hat{\mathbf{u}}_{m}^{(p)}\right\} d t=0 \\
(p=u, \ell ; m=1,2,3,4)
\end{array}
$$

where the matrix $\Lambda_{m}^{(p)}$ contains the Lagrange multiplier functions in the form

$$
\Lambda_{m}^{(p)}(t)=\left[\begin{array}{llll}
\Lambda_{n m}^{(p)}(t) & & & \\
& \Lambda_{t m}^{(p)}(t) & & \\
& & \Lambda_{z m}^{(p)}(t) & \\
& & & \Lambda_{z m}^{\prime(p)}(t)
\end{array}\right]
$$$$
(p=u, \ell ; m=1,2,3,4)
$$

The (unknown) Lagrange multiplier functions,$\Lambda_{\alpha m}^{(p)}(t)$, with $\alpha=n, t, z$, and $\Lambda_{z m}^{\prime(p)}(t)$, are assumed in polynomial forms as

$$
\begin{aligned}
& \left(\Lambda_{\alpha m}^{(p)}(t(\xi)), \Lambda_{z m}^{\prime(p)}(t(\xi))\right) \\
& =\sum_{j=0}^{J}\left(\lambda_{j(\alpha m)}^{(p)}, \lambda_{j(z m)}^{\prime(p)}\right) T_{j}\left(\xi ; \mathbf{t}_{\Gamma_{m}}^{(p)}, K\right) \\
& \quad(p=u, \ell ; m=1,2,3,4)
\end{aligned}
$$

where $T_{j}\left(\xi ; \mathbf{t}_{\Gamma_{m}}^{(p)}, K\right)$ represents the $j^{\text {th }}$ term of the $(K-1)^{\text {th }}$-degree B-spline functions defined along the straight boundary segment $\Gamma_{m}^{(p)}$, with $\mathbf{t}_{\Gamma_{m}}^{(p)}$ being the associated knot vector. Also, $\lambda_{j(\alpha m)}$, with $\alpha=n, t, z$, and $\lambda_{j(z m)}^{\prime}$ are the unknown Lagrange multipliers associated with each B-spline function, $T_{j}\left(\xi ; \mathbf{t}_{\Gamma_{m}}^{(p)}, K\right)$.

Substituting the expressions for the Lagrange multiplier functions from Eq. (46) into Eq. (45) and rearranging the terms, the constraint equations representing the prescribed displacements can be rewritten as

$$
\lambda_{m}^{(p)^{\mathrm{T}}}\left(\mathbf{C}_{m}^{(p)} \mathbf{q}^{(p)}-\mathbf{f}_{m c}^{(p)}\right)=0
$$

where

$$
\lambda_{m}^{(p)^{\mathrm{T}}}=\left\{\lambda_{1 m}^{(p)^{\mathrm{T}}}, \quad \lambda_{2 m}^{(p)^{\mathrm{T}}}, \cdots, \quad \lambda_{J m}^{(p)^{\mathrm{T}}}\right\}
$$

with

$$
\begin{aligned}
\lambda_{k m}^{(p)^{\mathrm{T}}} & =\left\{\begin{array}{llll}
\lambda_{k(n m)}^{(p)}, & \lambda_{k(t m)}^{(p)}, & \lambda_{k(z m)}^{(p)}, & \lambda_{k(z m)}^{(p)}
\end{array}\right\} \\
\mathbf{C}_{m}^{(p)^{\mathrm{T}}} & =\left[\begin{array}{llll}
\mathbf{C}_{1 m}^{(p)^{\mathrm{T}}} & \mathbf{C}_{2 m}^{(p)^{\mathrm{T}}} & \cdots & \mathbf{C}_{J m}^{(p)^{\mathrm{T}}}
\end{array}\right]
\end{aligned}
$$

with

$$
\mathbf{C}_{j m}^{(p)}=\int_{\Gamma_{m}^{(p)}} T_{j}\left(\xi ; \mathbf{t}_{\Gamma_{m}}^{(p)}, K\right) \mathbf{V}_{m}^{(p)^{\mathrm{T}}} d \Gamma
$$

and

$$
\mathbf{f}_{m c}^{(p)^{\mathrm{T}}}=\left\{\begin{array}{llll}
\mathbf{f}_{1(m c)}^{(p)^{\mathrm{T}}} & \mathbf{f}_{2(m c)}^{(p)^{\mathrm{T}}}, & \cdots, & \mathbf{f}_{J(m c)}^{(p)^{\mathrm{T}}}
\end{array}\right\}
$$

with

$$
\mathbf{f}_{j(m c)}^{(p)^{\mathrm{T}}}=\int_{\Gamma_{m}^{(p)}} T_{j}\left(\xi ; \mathbf{t}_{\Gamma_{m}}^{(p)}, K\right) \hat{\mathbf{u}}_{m}^{(p)^{\mathrm{T}}} d \Gamma
$$

for $(p=u, \ell ; m=1,2,3,4)$.

The constraint equations in Eq. (48) can be assembled to form a single matrix equation combining all of the constraint equations as

$$
\lambda^{(p)^{\mathrm{T}}}\left(\mathbf{C}^{(p)} \mathbf{q}^{(p)}-\mathbf{f}_{c}^{(p)}\right)=0 \quad(p=u, \ell)
$$

where

$$
\begin{aligned}
& \lambda^{(p)^{\mathrm{T}}}=\left\{\begin{array}{llll}
\lambda_{1}^{(p)^{\mathrm{T}}}, & \lambda_{2}^{(p)^{\mathrm{T}}}, & \cdots, & \lambda_{L}^{(p)^{\mathrm{T}}}
\end{array}\right\} \\
& \mathbf{C}^{(p)^{\mathrm{T}}}=\left[\begin{array}{llll}
\mathbf{C}_{1}^{(p)^{\mathrm{T}}} & \mathbf{C}_{2}^{(p)^{\mathrm{T}}} & \cdots & \mathbf{C}_{L}^{(p)^{\mathrm{T}}}
\end{array}\right]
\end{aligned}
$$




$$
\mathbf{f}_{c}^{(p)^{\mathrm{T}}}=\left\{\mathbf{f}_{1 c}^{(p)^{\mathrm{T}}}, \quad \mathbf{f}_{2 c}^{(p)^{\mathrm{T}}}, \cdots, \quad \mathbf{f}_{L c}^{(p)^{\mathrm{T}}}\right\}
$$

The system of constraint equations in Eq. (54) is unique, provided the rank of the matrix $\mathbf{C}^{(p)}$ is equal to the total number of constraint equations. Also, Eq. (54) can be treated as the potential energy of the reaction forces producing zero energy since $\mathbf{C}^{(p)} \mathbf{q}^{(p)}=\mathbf{0}$, and it can be referred to as the potential energy of the constraint forces, $V_{c}$, in the form

$$
V_{c}=\sum_{p=u, \ell} \lambda^{(p)^{\mathrm{T}}}\left(\mathbf{C}^{(p)} \mathbf{q}^{(p)}-\mathbf{f}_{c}^{(p)}\right)=0
$$

\section{Governing Equations}

The governing equations are derived based on the principle of virtual work

$$
\delta W_{i}=\delta W_{e}
$$

where $\delta W_{i}$ and $\delta W_{e}$ represent the virtual work due to internal and external forces, respectively, of the bonded single-lap joint.

The internal virtual work, $\delta W_{i}$, is the sum of the internal virtual work of the adherends and the adhesive, i.e.,

$$
\delta W_{i}=\delta W_{i}^{(u)}+\delta W_{i}^{(\ell)}+\delta W_{i}^{(a)}
$$

where the internal virtual work in the adherends and adhesive are expressed as

$$
\begin{aligned}
& \delta W_{i}^{(p)}=\int_{A_{p}} \delta \mathbf{e}^{(p)^{\mathrm{T}}} \mathbf{s}^{(p)} d A=\int_{A_{p}} \delta \mathbf{e}^{(p)^{\mathrm{T}}} \mathbf{E}^{(p)} \mathbf{e}^{(p)} d A \\
& \delta W_{i}^{(a)}=\int_{A_{a}} \delta \boldsymbol{\varepsilon}^{(a)^{\mathrm{T}}} \mathbf{s}^{(a)} d A=\int_{A_{a}} \delta \boldsymbol{\varepsilon}^{(a)^{\mathrm{T}}} \mathbf{E}^{(a)} \boldsymbol{\varepsilon}^{(a)} d A
\end{aligned}
$$

where $A_{p}$ denotes the areas of the adherends $(p=u, \ell)$ and adhesive $(p=a)$.

Substituting from Eqs. (24) and (27) and with the property of $\delta\left[\mathbf{L}_{N}^{(p)}\left(\mathbf{q}^{(p)}\right)\right] \mathbf{q}^{(p)}=\mathbf{L}_{N}^{(p)}\left(\mathbf{q}^{(p)}\right) \delta \mathbf{q}^{(p)}$, the total virtual strain vectors, $\delta \boldsymbol{\varepsilon}^{(a)}$ and $\delta \mathbf{e}^{(p)}$, are obtained as

$$
\delta \boldsymbol{\varepsilon}^{(a)}=\delta \mathbf{q}^{(u)^{\mathrm{T}}} \mathbf{L}_{a}^{(u)}-\delta \mathbf{q}^{(\ell)} \mathbf{L}_{a}^{(\ell)^{\mathrm{T}}}
$$

and

$$
\begin{aligned}
\delta \mathbf{e}^{(p)} & =\left[\mathbf{L}_{L}^{(p)}+2 \mathbf{L}_{N}^{(p)}\left(\mathbf{q}^{(p)}\right)\right] \delta \mathbf{q}^{(p)} \\
& ={ }^{*} \mathbf{H}^{(p)}\left(\mathbf{q}^{(p)}\right) \delta \mathbf{q}^{(p)}
\end{aligned}
$$

where

$$
{ }^{*} \mathbf{H}^{(p)}\left(\mathbf{q}^{(p)}\right)=\mathbf{L}_{L}^{(p)}+2 \mathbf{L}_{N}^{(p)}\left(\mathbf{q}^{(p)}\right)
$$

with $p=u, \ell$. The external virtual work is expressed as the sum of the virtual work due to externally applied forces, $\delta W_{e}^{(p)}$, and that arising from the boundary reaction forces, $\delta W_{c}^{(p)}$, i.e.,

$$
\delta W_{e}=\sum_{p=u, \ell} \delta W_{e}^{(p)}+\delta W_{c}^{(p)}
$$

The virtual work due to externally applied forces, $\delta W_{e}^{(p)}$, can be expressed in matrix notation as

$$
\delta W_{e}^{(p)}=\delta \mathbf{q}^{(p) T} \mathbf{p}^{(p)}
$$

in which

$$
\mathbf{p}^{(p) T}=\left\{\mathbf{p}_{\varepsilon}^{(p) T}, \mathbf{p}_{\kappa}^{(p) T}\right\}
$$

with

$$
\begin{aligned}
& \mathbf{p}_{\varepsilon}^{(p)}=\int_{\Gamma_{p}}\left\{\mathbf{V}_{x}^{(p)} t_{x}+\mathbf{V}_{y}^{(p)} t_{y}\right\} d \Gamma \\
& \mathbf{p}_{\kappa}^{(p)}=\int_{\Gamma^{(p)}}\left\{\mathbf{V}_{z}^{(p)} p_{z}+\mathbf{V}_{z, x}^{(p)} m_{x}+\mathbf{V}_{z, y}^{(p)} m_{y}\right\} d \Gamma
\end{aligned}
$$

The virtual work due to the boundary reaction forces, $\delta W_{c}^{(p)}$, is identical to the first variation of the potential energy expression in Eq. (56) as

$$
\begin{aligned}
\delta W_{c}^{(p)} & =-\delta V_{c} \\
& =-\delta \boldsymbol{\lambda}^{(p)^{\mathrm{T}}}\left(\mathbf{C}^{(p)} \mathbf{q}^{(p)}-\mathbf{f}_{c}^{(p)}\right) \\
& -\delta \mathbf{q}^{(p)^{T}} \mathbf{C}^{(p)^{T}} \lambda^{(p)}
\end{aligned}
$$

The virtual work due to the boundary reactions (constraint conditions) can be interpreted as the virtual work of the constraint forces, $\lambda^{(p)}$, over the virtual displacements, $\delta\left(\mathbf{C}^{(p)} \mathbf{q}^{(p)}\right)$, of the adherends and the virtual work of the constrained displacements (boundary conditions), $\mathbf{C}^{(p)} \mathbf{q}^{(p)}-\mathbf{f}_{c}^{(p)}$, over the virtual constraint forces, $\delta \lambda^{(p)}$. Although the term inside the parentheses in Eq. (67) is identical to zero, it is included in the virtual work expression in order to obtain a complete set of equations that contains both equilibrium equations and constraint conditions (kinematic 
boundary conditions) along the boundary of the adherends.

Substituting from Eqs. (59), (60), (64) and (67) while invoking the strain vectors, $\mathbf{e}^{(p)}(p=u, \ell)$ and $\boldsymbol{\varepsilon}^{(a)}$, from Eqs. (24) and (27), and their virtual forms, $\delta \mathbf{e}^{(p)}(p=u, \ell)$ and $\delta \boldsymbol{\varepsilon}^{(a)}$, from Eqs. (61) and (62), and rearranging the terms, the virtual work expression of Eq. (57) can be rewritten as

$$
\begin{aligned}
\delta \mathbf{q}^{(\ell)^{\mathrm{T}}} & \left(\mathbf{K}^{(\ell)}\left(\mathbf{q}^{(\ell)}\right)+\mathbf{K}_{11}^{(a)}\left(\mathbf{q}^{(\ell)}, \mathbf{q}^{(u)}\right)\right) \mathbf{q}^{(\ell)} \\
& +\delta \mathbf{q}^{(u)^{\mathrm{T}}}\left(\mathbf{K}^{(u)}\left(\mathbf{q}^{(u)}\right)+\mathbf{K}_{22}^{(a)}\left(\mathbf{q}^{(\ell)}, \mathbf{q}^{(u)}\right)\right) \mathbf{q}^{(u)} \\
& -\delta \mathbf{q}^{(u)^{\mathrm{T}}} \mathbf{K}_{21}^{(a)}\left(\mathbf{q}^{(\ell)}, \mathbf{q}^{(u)}\right) \mathbf{q}^{(\ell)} \\
& -\delta \mathbf{q}^{(\ell)^{\mathrm{T}}} \mathbf{K}_{12}^{(a)}\left(\mathbf{q}^{(\ell)}, \mathbf{q}^{(u)}\right) \mathbf{q}^{(u)} \\
& =\sum_{p=u, \ell} \delta \mathbf{q}^{(p)^{\mathrm{T}}} \mathbf{p}^{(p)}-\delta \lambda^{(p)^{\mathrm{T}}} \mathbf{C}^{(p)} \mathbf{q}^{(p)} \\
& -\delta \mathbf{q}^{(p)^{\mathrm{T}}} \mathbf{C}^{(p)^{\mathrm{T}}} \lambda^{(p)}+\delta \lambda^{(p)^{\mathrm{T}}} \mathbf{f}_{c}^{(p)}
\end{aligned}
$$

where

$$
\begin{gathered}
\mathbf{K}^{(p)}\left(\mathbf{q}^{(p)}\right)=\int_{A_{p}}{ }^{*} \mathbf{H}^{(p)^{\mathrm{T}}}\left(\mathbf{q}^{(p)}\right) \mathbf{E}^{(p)} \mathbf{H}^{(p)}\left(\mathbf{q}^{(p)}\right) d A \\
(p=u, \ell) \\
\mathbf{K}_{\alpha \beta}^{(a)}\left(\mathbf{q}^{(u)}, \mathbf{q}^{(\ell)}\right)=\int_{A_{a}} \mathbf{L}_{a}^{(\alpha)^{\mathrm{T}}} \mathbf{E}^{(a)}\left(\mathbf{q}^{(u)}, \mathbf{q}^{(\ell)}\right) \mathbf{L}_{a}^{(\beta)} d A \\
(\alpha, \beta=1,2)
\end{gathered}
$$

For arbitrary variations of the virtual solution vectors $\delta \mathbf{q}^{(p)}$ and $\delta \lambda^{(p)}(p=u, \ell)$, Eq. (68) can further be rearranged and put into a more compact form

$$
\begin{aligned}
& {\left[\begin{array}{cccc}
\mathbf{K}_{11}\left(\mathbf{q}^{(u)}, \mathbf{q}^{(\ell)}\right) & \mathbf{K}_{12}\left(\mathbf{q}^{(u)}, \mathbf{q}^{(\ell)}\right) & \mathbf{C}^{(\ell) \mathrm{T}} & \mathbf{0} \\
\mathbf{K}_{12}^{\mathrm{T}}\left(\mathbf{q}^{(u)}, \mathbf{q}^{(\ell)}\right) & \mathbf{K}_{22}^{\mathrm{T}}\left(\mathbf{q}^{(u)}, \mathbf{q}^{(\ell)}\right) & \mathbf{0} & \mathbf{C}^{(u) \mathrm{T}} \\
\mathbf{C}^{(\ell)} & \mathbf{0} & \mathbf{0} & \mathbf{0} \\
\mathbf{0} & \mathbf{C}^{(u)} & \mathbf{0} & \mathbf{0}
\end{array}\right]} \\
& \times\left\{\begin{array}{c}
\mathbf{q}^{(\ell)} \\
\mathbf{q}^{(u)} \\
\lambda^{(\ell)} \\
\lambda^{(u)}
\end{array}\right\}=\left\{\begin{array}{c}
\mathbf{p}^{(\ell)} \\
\mathbf{p}^{(u)} \\
\mathbf{f}_{c}^{(\ell)} \\
\mathbf{f}_{c}^{(u)}
\end{array}\right\}
\end{aligned}
$$

where

$$
\mathbf{K}_{11}\left(\mathbf{q}^{(u)}, \mathbf{q}^{(\ell)}\right)=\mathbf{K}^{(\ell)}\left(\mathbf{q}^{(\ell)}\right)+\mathbf{K}_{11}^{(a)}\left(\mathbf{q}^{(u)}, \mathbf{q}^{(\ell)}\right)
$$

$$
\begin{aligned}
\mathbf{K}_{22}\left(\mathbf{q}^{(u)}, \mathbf{q}^{(\ell)}\right) & =\mathbf{K}^{(u)}\left(\mathbf{q}^{(u)}\right)+\mathbf{K}_{22}^{(a)}\left(\mathbf{q}^{(u)}, \mathbf{q}^{(\ell)}\right) \\
\mathbf{K}_{12}\left(\mathbf{q}^{(u)}, \mathbf{q}^{(\ell)}\right) & =-\mathbf{K}_{12}^{(a)}\left(\mathbf{q}^{(u)}, \mathbf{q}^{(\ell)}\right) \\
\mathbf{K}_{21}\left(\mathbf{q}^{(u)}, \mathbf{q}^{(\ell)}\right) & =-\mathbf{K}_{21}^{(a)}\left(\mathbf{q}^{(u)}, \mathbf{q}^{(\ell)}\right) \\
& =\mathbf{K}_{12}^{\mathrm{T}}\left(\mathbf{q}^{(u)}, \mathbf{q}^{(\ell)}\right)
\end{aligned}
$$

Note that the submatrices $\mathbf{K}_{s s}\left(\mathbf{q}^{(u)}, \mathbf{q}^{(\ell)}\right)$ and $\mathbf{K}_{p p}\left(\mathbf{q}^{(p)}, \mathbf{q}^{(s)}\right)$ are dependent on the unknown variables, $\mathbf{q}^{(u)}, \mathbf{q}^{(\ell)}$ rendering the governing equations nonlinear, and that these submatrices are nonsymmetric. The solution to this equation requires a nonlinear iterative solution technique that utilizes LU decomposition. Therefore, the Newton-Raphson iteration method in conjunction with Broyden's automatic Jacobian matrix update procedure is employed. The iterative solution procedure begins with the initial guess of the incremental unknown variables. The initial guess is obtained from the linearized equilibrium equations, and the converged solution is achieved through incremental corrections to the initial guess.

\section{Numerical Results}

The present approach is first validated against the nonlinear finite element solution of isotropic single-lap joints with linear and bilinear adhesive behaviors. As described in Fig. 1, the adherends have identical planar geometries, with the width and length dimensions specified as $W^{(u)}=W^{(\ell)}=W=20 \mathrm{~mm}$ and $L^{(u)}=L^{(\ell)}=$ $L=60 \mathrm{~mm}$, respectively. The overlap length of the joint is given as $L^{(a)}=20 \mathrm{~mm}$. The thicknesses of the adherends and the adhesive are specified as $h^{(u)}=h^{(\ell)}=h=1.5 \mathrm{~mm} \quad$ and $\quad h^{(a)}=0.2 \mathrm{~mm}$, respectively. These dimensions are the same as those considered previously by Edlund and Klarbring 9 .

The adherends are made of aluminum with Young's modulus and Poisson's ratio specified as $E^{(u)}=E^{(\ell)}=$ $E=70000 \mathrm{MPa}$ and $v^{(u)}=v^{(\ell)}=v=0.3$, respectively. Also, the adhesive exhibits either a linear or a bilinear elastic material behavior. In the case of linearly elastic material behavior, the shear modulus and Poisson's ratio of the adhesive are specified as $G^{(a)}=3,000 \mathrm{MPa}$ and $v^{(a)}=0.3$, respectively. In the case of bilinear material behavior, the material parameters of the adhesive are defined as $G_{1}^{(a)}=3,000 \mathrm{MPa}, G_{2}^{(a)}=$ $1,500 \mathrm{MPa}$, and $v^{(a)}=0.3$. The single-lap joint simply supported along the loaded ends is subjected to uniform tension of $N_{0}=150 \mathrm{~N} / \mathrm{mm}$ in 10 equal load increments. 
The present analysis results are compared against predictions obtained from a two-dimensional nonlinear finite element analysis (FEA) using ANSYS, a commercially available program. The scaled deformation of the bonded single-lap joint with linearly elastic adhesive behavior at the final load step is shown in Fig. 4.

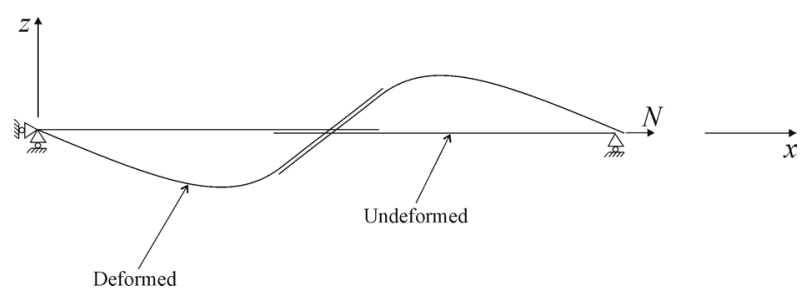

Fig. 4 The scaled deformation of the bonded lap joint made of isotropic adherends with linear adhesive behavior at the last load step $\left(N / N_{0}=1\right)$.

As observed, the bonded single-lap joint bends asymmetrically as it is stretched from the right edge of the lower adherend. The asymmetric deformation occurs primarily due to the presence of eccentric loading, boundary conditions, and the geometrical coupling between the adherends.

The transverse normal (peeling), $\sigma_{z z}$, and transverse shear stresses, $\sigma_{x z}$ and $\sigma_{y z}$, in the adhesive for the case of linearly elastic adhesive material behavior corresponding to the final load step is illustrated in Fig. 5. The steep variations of the peeling, $\sigma_{z z}$, and shearing stresses, $\sigma_{x z}$ and $\sigma_{y z}$, near the edges and corners of the adhesive are captured successfully by the present approach. Note that both peeling stress $\sigma_{z z}$ and shearing stress $\sigma_{x z}$ are symmetric along the horizontal and vertical centerlines of the adhesive whereas the shearing stress $\sigma_{y z}$ is asymmetrically distributed.

A comparison of the peeling stress $\sigma_{z z}$ and shearing stress $\sigma_{x z}$ evaluated along the horizontal centerline (i.e., along $y=10 \mathrm{~mm}$ ) from the present analysis with those of the FEA is shown in Fig. 6. The comparison indicates close agreement for both the linearly elastic and bilinear adhesive material behaviors. The present solution method captures the expected steep variation of the shearing and peeling stresses along the adhesive edges.

A comparison of the slope variation at the center of the overlap as the load is increased for the present solution method with that of the nonlinear FEA with ANSYS indicates close agreement, as shown in Fig. 7.
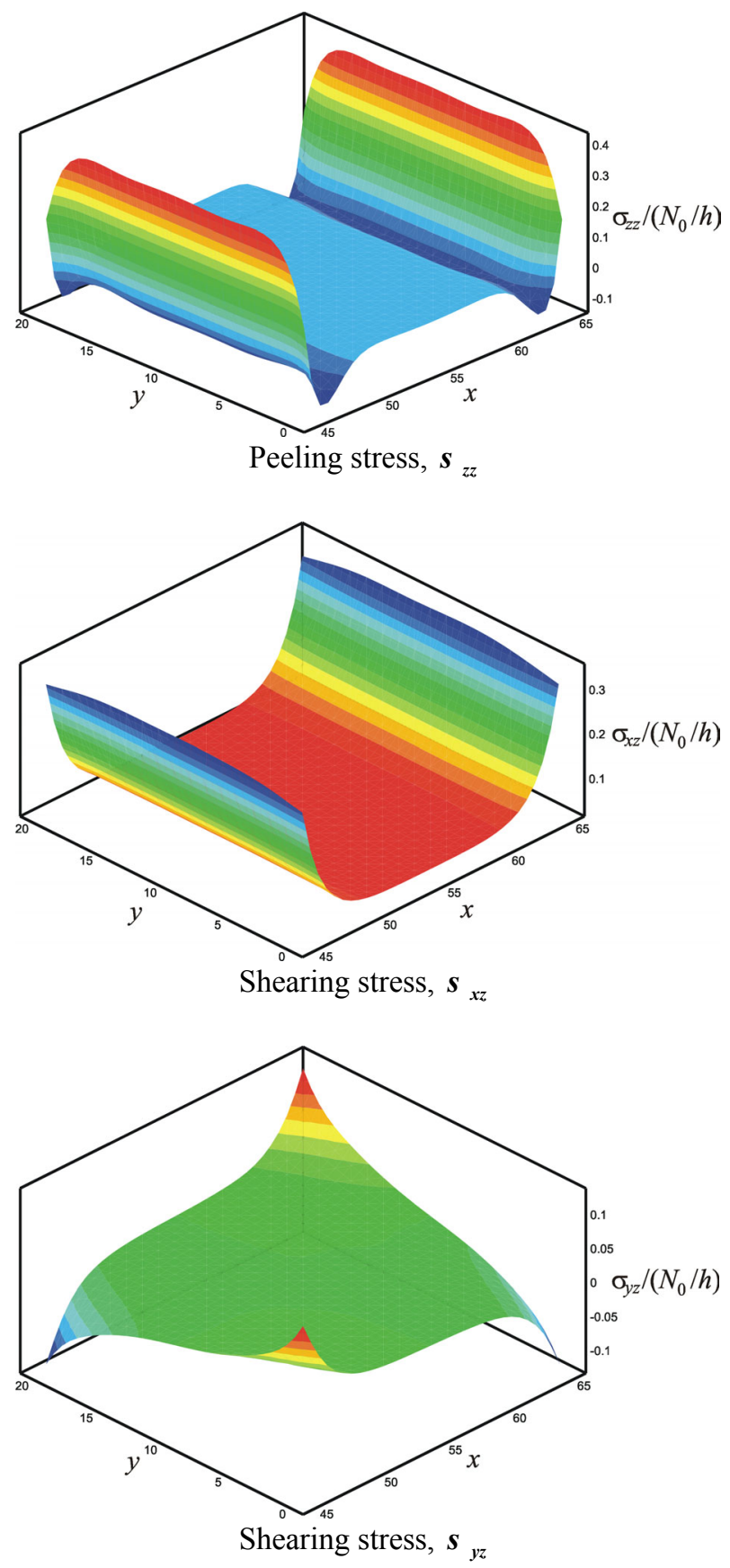

Fig. 5 Variation of the stress components in the adhesive: peeling stress, $s_{z z}$; shearing stress, $s_{x z}$; shearing stress, $s_{y z}$.

The small difference can be attributed to the modeling differences and the fact that FEA includes transverse shearing deformations in the adherends, as well as in the adhesive, whereas the present approach is based on the Kirchhoff plate theory, which excludes the transverse shear deformations in the adherends. 


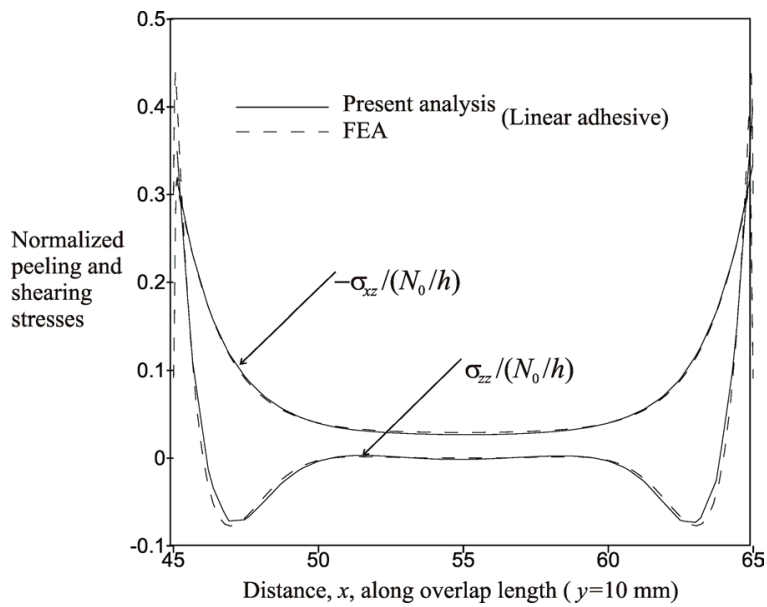

Linear adhesive

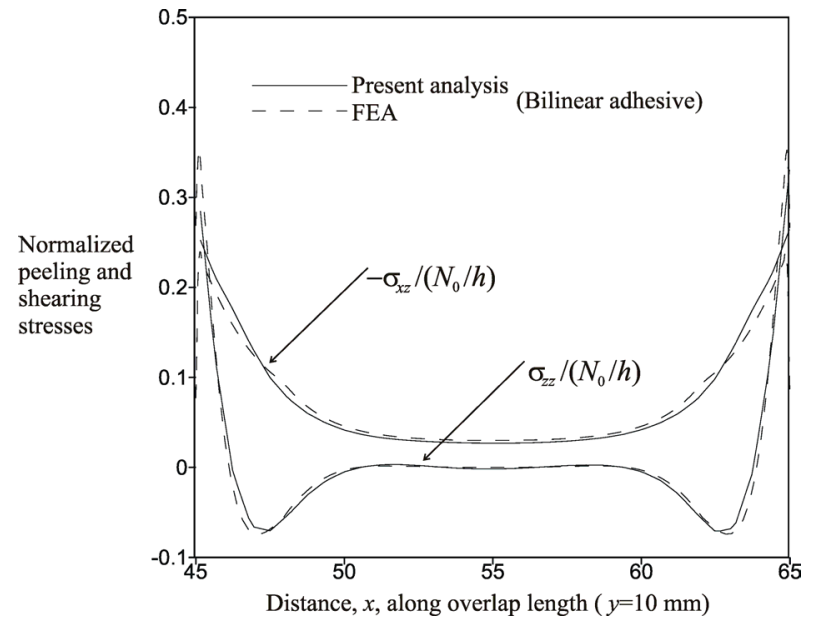

Bilinear adhesive.

Fig. 6 Comparison of the peeling stress, $s_{z z}$, and shearing stress, $s_{x z}$, along the horizontal centerline, between the present approach and the finite element analysis for: linear adhesive and bilinear adhesive.

The capability of the present approach is demonstrated by considering a bonded composite single-lap joint of angle-ply laminates (adherends) as shown in Fig. 1. The adherend length and width and the overlap length, as well as the loading and boundary conditions, are identical to those of the validation case except for the presence of tapered adherend edges. The lower and upper laminate edges are tapered (beveled) toward the adhesive edges, as shown in Fig. 1, where $\Delta^{(u)}$ and $\Delta^{(\ell)}$ indicate the taper lengths of the upper and lower adherends, respectively.

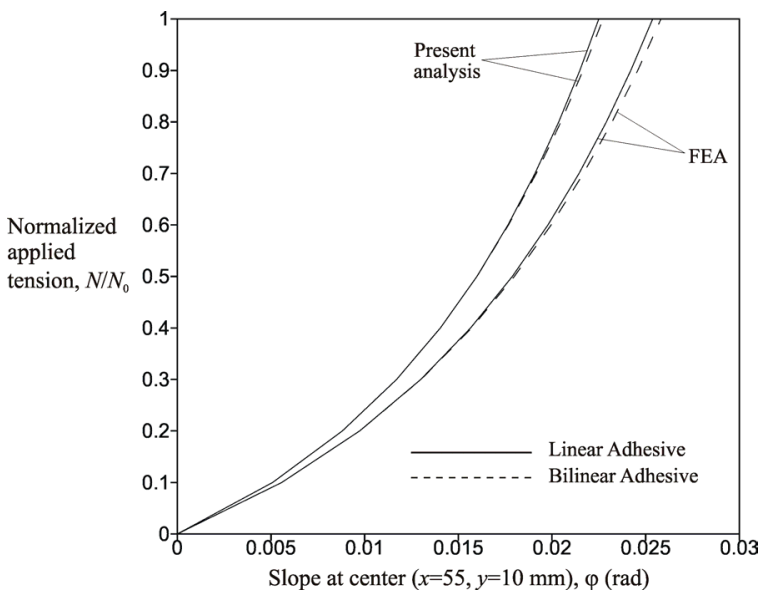

Fig. 7 Comparison of the rotation at the center of the overlap as a function of applied load between the present approach and the finite element analysis.

Both adherends are symmetrically laminated and their angle-ply stacking sequence is given by $[\theta /-\theta]_{4 s}$, where $\theta$ is referred to as the angle-ply laminate parameter. Each ply made of Graphite-Epoxy has properties of $E_{L}=127.56 \mathrm{GPa}, E_{T}=11.31 \mathrm{GPa}$, $v_{L T}=0.3$, and $G_{L T}=6 \mathrm{GPa}$, with equal nominal ply thicknesses of $t_{k}=0.0762 \mathrm{~mm}$. Hence, the total thickness of the untapered laminate becomes $2 h^{(u)}=2 h^{(\ell)}=2 h=1.2192 \mathrm{~mm}$. The thickness of the adhesive is specified as $2 h^{(a)}=0.12 \mathrm{~mm}$. The linearly elastic behavior of the adhesive is defined by a shear modulus of $G_{1}^{(a)}=G_{2}^{(a)}=G^{(a)}=0.4147 \mathrm{GPa}$, and the bilinear adhesive material behavior is defined by the parameters $G_{1}^{(a)}=0.4147 \mathrm{GPa}$ and $G_{2}^{(a)}=300 \mathrm{GPa}$ with a characteristic shear strain of $\gamma_{c}^{(a)}=0.03$.

The effects of the tapered adherend geometry and the angle-ply laminate parameter, $\theta$, on the geometrically nonlinear behavior of the bonded lap joint are investigated by (1) varying the taper lengths, $\Delta^{(u)}$ and $\Delta^{(l)}$, from 0 to $5 \mathrm{~mm}$ in five equal increments while specifying the value of $\theta=45$ degrees (i.e, $[+45 /-45]_{4 s}$ laminate), and (2) by varying the angle-ply laminate parameter, $\theta$, as $0,5,10,15,30$ and 45 degrees while assuming an untapered adherend edge, i.e., $\Delta^{(u)}=\Delta^{(l)}=0$.

The effects of taper lengths on the transverse normal stress, $\sigma_{z z}$, and the transverse shear stress, $\sigma_{x z}$, both evaluated along the horizontal centerline $y=10 \mathrm{~mm}$, are depicted in Figs. 8 and 9, respectively, for both linearly elastic and bilinear adhesive material behaviors. As shown in these figures, the peeling stress, 


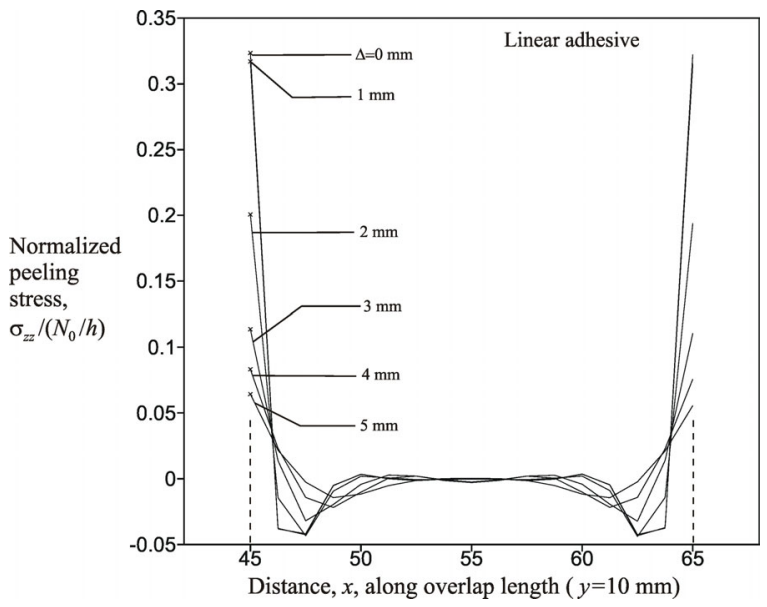

Linearly elastic adhesive

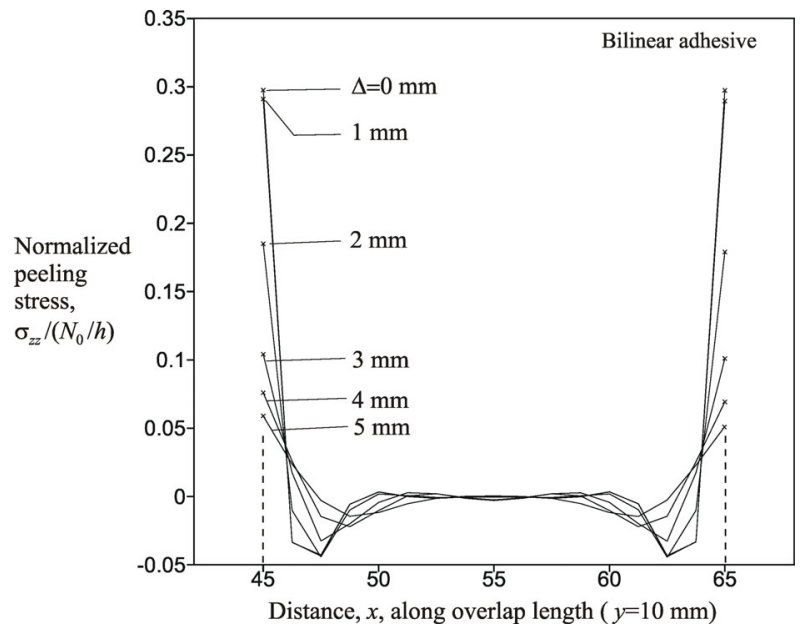

Bilinear elastic adhesive

Fig. 8 Variation of the peeling stress, $s_{z z}$, along the horizontal centerline for varying adherend taper lengths: linearly elastic and bilinear adhesive behavior.

$\sigma_{z z}$, reduces with increasing taper length, and the shearing stress, $\sigma_{x z}$, reduces slightly along the horizontal centerline of the adhesive. The transverse shear stress, $\sigma_{y z}$, evaluated along the vertical centerline of $x=45 \mathrm{~mm}$ is depicted in Fig. 10, also for both linearly elastic and bilinear adhesive material behaviors. The shear stress component near the corners of the adhesive edges decreases significantly with increasing taper length. Although not shown here, the transverse shearing stress, $\sigma_{x z}$, also reduces significantly at the corners of the adhesive edges as a result of increasing taper length. As observed in Figs. 8-
10 , the bilinear adhesive material behavior yields stress variations identical to those of the linear adhesive behavior, but with relatively lower transverse normal and shear stress variations than those of the linearly elastic adhesive material.

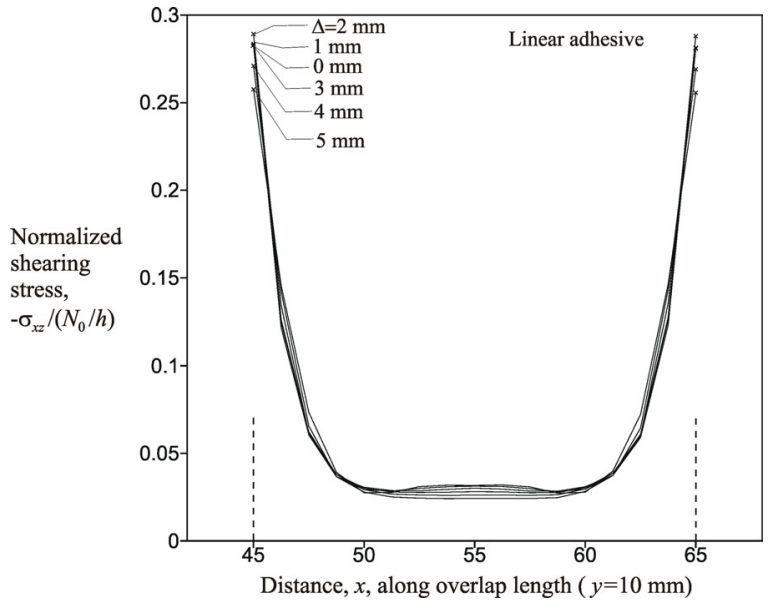

Linearly elastic adhesive

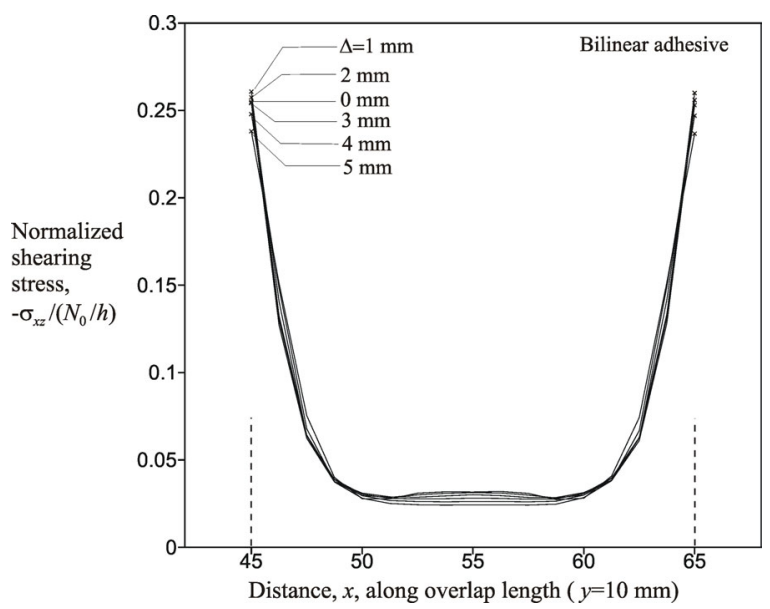

Bilinear elastic adhesive

Fig. 9 Variation of the shearing stress, $s_{x z}$, along the horizontal centerline for varying adherend taper lengths: linearly elastic and bilinear adhesive behavior.

Variations of the peeling stress, $\sigma_{z z}$, and transverse shear stresses, $\sigma_{x z}$ and $\sigma_{y z}$, at the final load step, $N_{0} / N_{0}^{\max }=1.0$, in the adhesive with linearly elastic adhesive material in the absence of tapered adherend edges are illustrated in Fig. 11. As expected, both the 


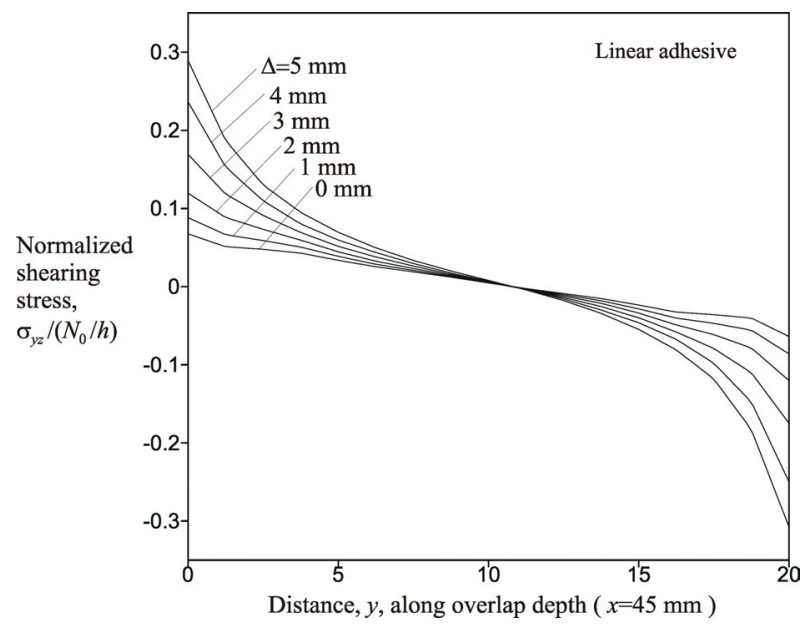

Linearly elastic adhesive

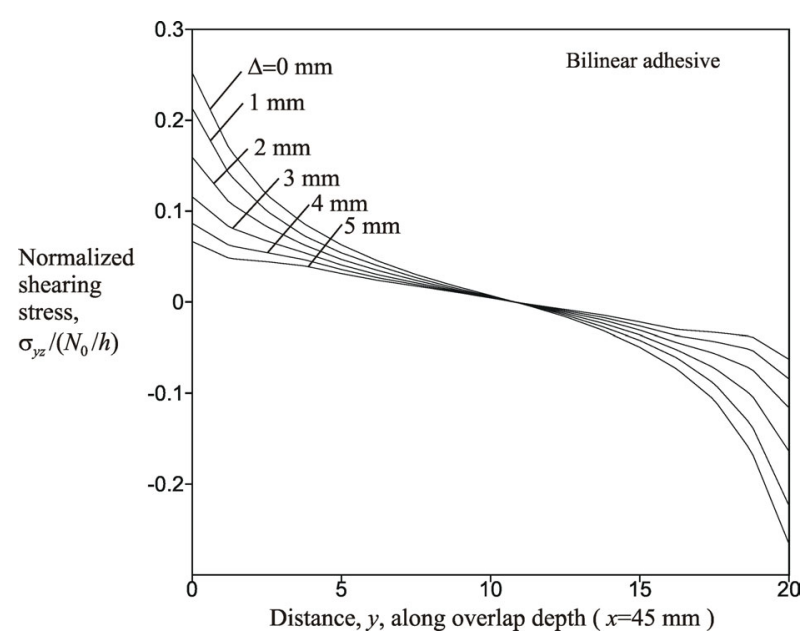

Bilinear elastic adhesive

Fig. 10 Variation of the shearing stress, $s_{y z}$, along the vertical centerline for varying adherend taper lengths: linearly elastic and bilinear adhesive behavior.

transverse shear and peeling stresses increase rapidly near the edges of the adhesive. As observed in Figure 11 , all of the adhesive stress variations are asymmetric with respect to the horizontal and vertical centerlines.

The adherends are symmetric angle-ply laminates with no material coupling between stretching and bending that may cause twisting in the adherends and result in asymmetric stress distribution in the adhesive. However, the coupling between the bending and twisting deformations disturbs the symmetry in the adhesive stresses.
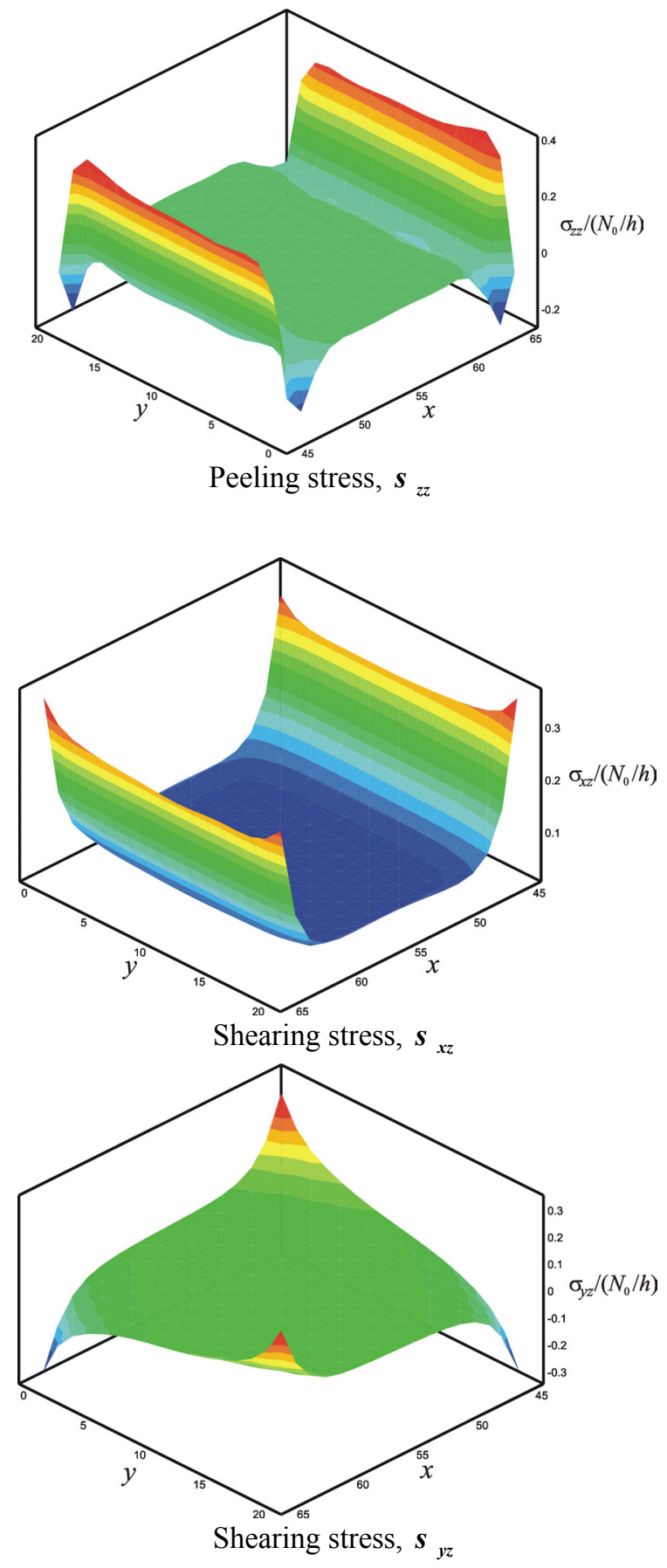

Fig. 11 Variation of the stress components in the adhesive: peeling stress, $s_{z z}$, shearing stress, $s_{x z}$ along the horizontal vertical centerline, and shearing stress, $s_{y z}$ for untapered adherends of $[ \pm 45]_{4 s}$ angle-ply laminates with linear elastic adhesive behavior. 
In order to understand this behavior, the adhesive stresses are evaluated along the left edge of the adhesive (i.e., $x=45$ ) for varying angle-ply laminate parameter, $\theta$, as shown in Fig. 12.

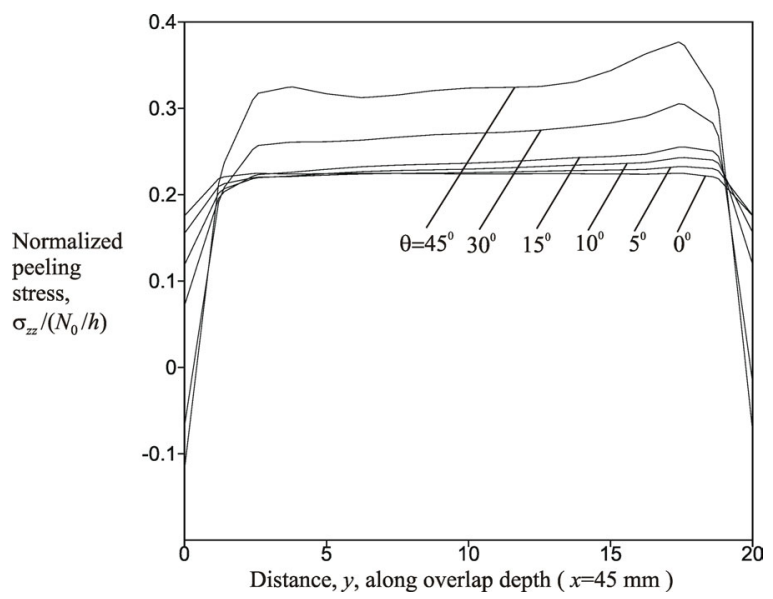

Peeling stress

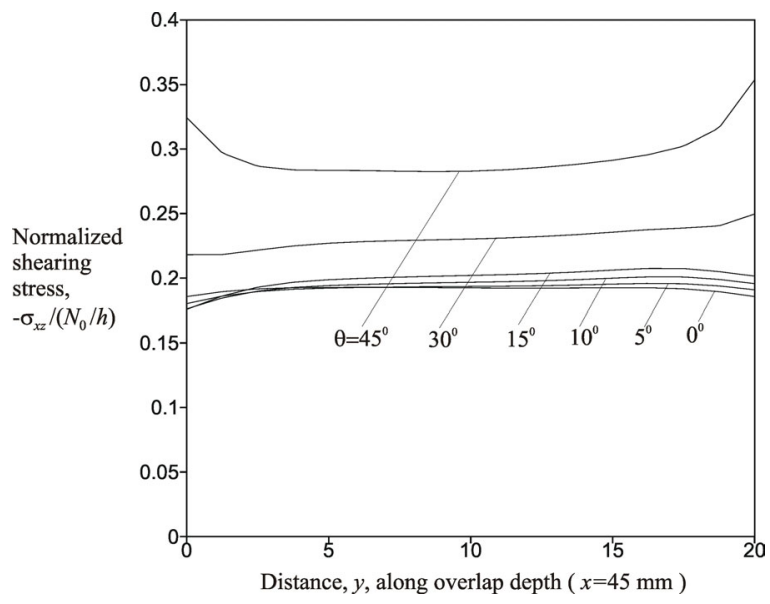

Shearing stress

Fig. 12 Variation of the stress components in the adhesive: peeling stress, $\boldsymbol{s}_{z z}$ and shearing stress, $s_{x z}$ along the left edge of the adhesive for varying values of angle-ply parameter, $q$.

These results are obtained for untapered adherends and linearly elastic adhesive behavior. As shown in Fig. $12 \mathrm{a}$, the peeling stress, $\sigma_{z z}$, is symmetric along the adhesive edge for $\theta=0$. As $\theta$ is gradually increased from 0 to 15 degrees, the peeling stress near the upper corner of the left adhesive edge (i.e., $x=45$ and $y=20$ ) becomes higher than that near the lower corner (i.e., $x=$ 45 and $y=0$ ). As $\theta$ is further increased, the difference in the peeling stress near the corners of the adhesive edge becomes more pronounced, as shown in Fig. 12a. A similar behavior is also observed in the transverse shearing stress, $\sigma_{x z}$, where the magnitude of stress at one corner is different than that at the other corner, as shown in Fig. 12b. Both the peeling and shearing stresses increase with increasing $\theta$. This is primarily due to the reduction of adherend in-plane and bending stiffnesses in the longitudinal direction. The lower the in-plane and bending stiffnesses of the adherends the higher the edge effects.

\section{Conclusions}

A semi-analytical solution procedure was presented for the geometrically nonlinear three-dimensional analysis of a single-lap joint made of composite tapered adherends bonded by a linearly elastic or bilinear adhesive. The nonlinear equations of equilibrium were derived based on the principle of virtual displacements in conjunction with the von Karman nonlinear plate theory for the adherends and the shear-lag theory for the adhesive. The bilinear adhesive material behavior was incorporated by computing the material parameters from an effective shear stress-shear strain relationship. The displacement fields were assumed in the form of a double series containing fifth-order B-spline functions (displacement modes) in each direction. The resulting nonlinear equations of equilibrium were then solved numerically by employing the Newton-Raphson incremental iterative procedure along with Broyden's automatic Jacobian matrix update.

A comparison against a two-dimensional nonlinear FEA solution established the capability of the present approach to accurately capture the steep variations of both peeling and shearing stresses in the vicinity of the adhesive edges, as well as at the corners. Furthermore, the variation of overlap rotations indicates that the stress-induced effects due to geometric nonlinearity were captured by preserving the nonlinear terms in the strain displacement relations.

In the case of a bonded single-lap joint of angle-ply laminates, the tapered edges led to a considerable reduction of the peeling stresses and a slight reduction of the shearing stress component in the longitudinal direction $\left(\sigma_{x z}\right)$. However, the increase in taper length reduced all the adhesive stress components at the corners of the adhesive region. Furthermore, the increase in the angle-ply laminate parameter not only increased the stress concentration near the adhesive edges but also changed the symmetric distribution of peeling and longitudinal (transverse) shearing stresses to asymmetric due to the presence of material coupling 
between bending and twisting deformations of the angle-ply laminates.

This special-purpose three-dimensional analysis method accurately predicts the geometrically nonlinear behavior of a bonded single-lap, tapered, composite joint subjected to uniaxial tension while accounting for the presence of tapering of the adherend thickness, changes in the overlap length of the adherends, and the linear and bilinear elastic behaviors of the adhesive.

\section{Appendix}

The B-spline functions, $T_{i}\left(\alpha ; \mathbf{t}_{\alpha}^{(p)}, K\right)$, with $\alpha=x, y$ and $p=\ell, u$, employed in Eq. (8) are defined recursively in the form ${ }^{13}$

$$
\begin{aligned}
T_{i}\left(\alpha ; \mathbf{t}_{\alpha}^{(p)}, K\right) & =\frac{\left(\alpha-t_{\alpha(i)}^{(p)}\right)}{\left(t_{\alpha(i+K-1)}^{(p)}-t_{\alpha(i)}^{(p)}\right)} T_{i}\left(\alpha ; \mathbf{t}_{\alpha}^{(p)}, K-1\right) \\
& +\frac{\left(t_{\alpha(i+K)}^{(p)}-\alpha\right)}{\left(t_{\alpha(i+K)}^{(p)}-t_{\alpha(i+1)}^{(p)}\right)} T_{i+1}\left(\alpha ; \mathbf{t}_{\alpha}^{(p)}, K-1\right)
\end{aligned}
$$

with $i=1,2, \ldots, M_{\alpha}^{(p)}$ and $k>1$. The variable $t_{\alpha(i)}^{(p)}$ represents the components of the knot vector, $\mathbf{t}_{\alpha}^{(p)}$, for the $(K-1)^{\text {st }}$-degree B-spline functions. The knot vector, $\mathbf{t}_{\alpha}^{(p)}$, is defined in terms of the Cartesian coordinate $(\alpha=x, y)$ of the selected points as

$$
\begin{aligned}
\mathbf{t}_{\alpha}^{(p)}= & \left\{t_{\alpha(0)}^{(p)}, t_{\alpha(1)}^{(p)}, \ldots, t_{\alpha\left(N_{\alpha}^{(d)}\right)}^{(p)}, t_{\alpha\left(N_{\alpha}^{(d)}+1\right)}^{(p)},\right. \\
& \left.t_{\alpha\left(N_{\alpha}^{(d)}+2\right)}^{(p)}, \ldots, t_{\alpha\left(N_{\alpha}^{(d)}+2 K\right)}^{(p)}\right\} \\
= & \left\{\alpha_{0}^{(p)}, \alpha_{0}^{(p)}, \ldots, \alpha_{0}^{(p)}, \alpha_{1}^{(p)}, \alpha_{2}^{(p)}, \ldots,\right. \\
& \left.\alpha_{N_{\alpha}^{(p)}}^{(p)}, \alpha_{N_{\alpha}^{(P)}+1}^{(p)}, \alpha_{N_{\alpha}^{(p)}+1}^{(p)}, \ldots, \alpha_{N_{\alpha}^{(p)}+1}^{(p)}\right\}
\end{aligned}
$$

where $N_{\alpha}^{(p)}+1(\alpha=x, y ; p=u, \ell)$ denotes the number of distinct knot points selected along the $\alpha$ direction in the lower and upper adherends. Based on the definition of the knot vector, $\mathbf{t}_{\alpha}^{(P)}$, the end points (i.e., $\alpha_{\alpha}^{(p)}$ and $\left.\alpha_{N_{\alpha}^{(P)}+1}^{(p)}\right)$ are repeated $K$ times. In Eqs. (72) and (73), the relationship between the number of knot points and the extent of the B-spline functions, $M_{\alpha}^{(p)}$, is given as

$$
N_{\alpha}^{(p)}=M_{\alpha}^{(p)}-K+3
$$

In this study, the B-spline functions with $K=5$ are chosen to have 20 and 7 knot points in the $x$ - and $y$ - directions, respectively. The knot vectors are defined as

$$
\begin{aligned}
\mathbf{t}_{x}^{(l)}= & \{45,45,45,45,45,46,47,49,52,55,58, \\
& 61,63,64,64.5,65,66,69,74,80,87.5, \\
& 95,101,106,10,110,110,110,110\} \\
\mathbf{t}_{x}^{(u)}= & \{0,0,0,0,0,4,9,15,22.5,30,36,41,44, \\
& 45,46,47,49,52,55,58,61,63,64,64.5, \\
& 65,65,65,65,65\} \\
\mathbf{t}_{y}^{(l)}= & \mathbf{t}_{y}^{(u)}=\{0,0,0,0,0,3.33,6.66,10,13.33, \\
& 16.66,20,20,20,20,20\}
\end{aligned}
$$

This results in a B-spline series representation of the displacement components (i.e., $u_{x}^{(p)}, u_{y}^{(p)}$, and $u_{z}^{(p)}$, with $p=l, u)$ with the series terminating at $M_{x}^{(p)}=23$ and $M_{y}^{(p)}=9$.

\section{$\underline{\text { References }}$}

${ }^{1}$ Tsai, M. Y., and Morton, J., "An Evaluation of Analytical and Numerical Solutions to the Single-Lap Joint," International Journal of Solids and Structures, Vol. 31, 1994, pp. 2537-2563.

${ }^{2}$ Ding, S., and Kumosa, M., "Singular Stress Behavior at an Adhesive Interface Corner," Engineering Fracture Mechanics, Vol. 47, 1994, pp. 503-519.

${ }^{3}$ Osnes, H., and Andersen, A., "Computational Analysis of Geometric Nonlinear Effects in Adhesively Bonded Single Lap Composite Joints," Composites: Part B, Vol. 34, 2003, pp. 417-427.

${ }^{4}$ Dattaguru, B., Everett, R. A., Jr., Whitcomb, J. D., and Johnson, W. S., "Geometrically Nonlinear Analysis of Adhesively Bonded Joints," Journal of Engineering Materials and Technology, Vol. 106, 1984, pp. 59-65.

${ }^{5}$ Reddy, J. N., and Roy, S., "Non-Linear Analysis of Adhesively Bonded Joints," International Journal of Non-Linear Mechanics, Vol. 23, 1988, pp. 97-112.

${ }^{6}$ Pandey, P. C., Shankaragouda, H., and Singh, A. K., "Nonlinear Analysis of Adhesively Bonded Lap Joints Considering Viscoplasticity in Adhesives," Computers and Structures, Vol. 70, 1999, pp. 387-413.

${ }^{7}$ Apalak, M. K., and Gunes, R., "On Non-Linear Thermal Stresses in an Adhesively Bonded Single Lap Joints," Computers and Structures, Vol. 80, 2001, pp. 85-98.

${ }^{8}$ Penado, F. E., "A Simplified Method for the Geometrically Nonlinear Analysis of the Single-Lap Joint," Journal of Thermoplastic Composite Materials, Vol. 11, 1998, pp. 272-287.

${ }^{9}$ Goland, M., and Reissner, E., "The Stresses in Cemented Joints," Journal of Applied Mechanics, Vol. 66, 1944, pp. A17-A27. 
${ }^{10}$ Edlund, U., and Klarbring, A., "A Geometrically Nonlinear Model of the Adhesive Joint Problem and Its Numerical Treatment," Computer Methods in Applied Mechanical Engineering, Vol. 96, 1992, pp. 329-350.

${ }^{11}$ Pandey, P. C., and Narasimhan, S., "Three-Dimensional Nonlinear Analysis of Adhesively Bonded Lap Joints Considering Viscoplasticity in Adhesives," Computers and Structures, 2001, Vol. 79, pp. 769-783.

${ }^{12}$ Narasimhan, S., and Pandey, P. C., "Three-Dimensional Material and Geometrical Nonlinear Analysis of Adhesively Bonded Single-Lap Joints," Defense Science Journal, 2003, Vol. 53, pp. 175-188.
${ }^{13}$ Hoschek, J., and Lasser, D., Fundamentals of Computer Aided Geometric Design, A K Peters, LTD, Massachusetts, 1993.

${ }^{14}$ Fung, Y. C., and Tong, P., Classical and Computational Solid Mechanics, World Scientific Publishing Co. Pte. Ltd., Singapore, 2001.

${ }^{15}$ Geradin, M., Idelsohn, S., and Hogge, M., "Computational Strategies for the Solution of Large Nonlinear Problems via Quasi-Newton Methods," Computers and Structures, Vol. 13, 1981, pp. 73-81. 